\title{
On the Lagrangian and Hamiltonian aspects of infinite -dimensional dynamical systems and their finite-dimensional reductions
}

\author{
YA. Prykarpatsky and A.M. Samoilenko
}

*) Institute of Mathematics at the NAS, Kiev 01601, Ukraine, and the AGH

University of Science and Technology, Department of Applied Mathematics,

Krakow30059 Poland (yarprybnl.gov, sam@imath.kiev.ua)

15 March 2004

\begin{abstract}
A description of Lagrangian and Hamiltonian formalisms naturally arisen from the invariance structure of given nonlinear dynamical systems on the infinitedimensional functional manifold is presented. The basic ideas used to formulate the canonical symplectic structure are borrowed from the Cartan's theory of differential systems on associated jet-manifolds. The symmetry structure reduced on the invariant submanifolds of critical points of some nonlocal Euler-Lagrange functional is described thoroughly for both differential and differential discrete dynamical systems. The Hamiltonian representation for a hierarchy of Lax type equations on a dual space to the Lie algebra of integral-differential operators with matrix coefficients, extended by evolutions for eigenfunctions and adjoint eigenfunctions of the corresponding spectral problems, is obtained via some special Backlund transformation. The connection of this hierarchy with integrable by Lax spatially two-dimensional systems is studied.
\end{abstract}

\section{Introduction}

One of the fundamental problems in modern theory of infinite-dimensional dynamical systems is that of an invariant reduction them upon some invariant submanifolds with enough rich mathematical structures to treat their properties analytically. The first approaches to these problems were suggested still at the late times of the preceding century, in the classical oeuvres by S.Lie, J.Liouville, J.Lagrange, V.R.Hamilton, J.Poisson and E.Cartan. They introduced at first the important concepts of symmetry, conservation law, symplectic, Poisson and Hamiltonian structures as well invariant reduction procedure, which appeared to be extremely useful for proceeding studies. These notions were widely generalized further by

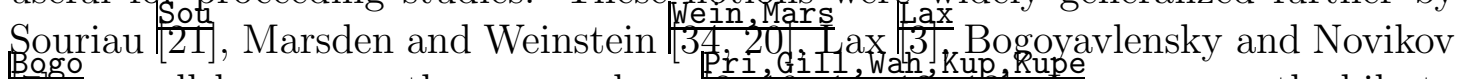

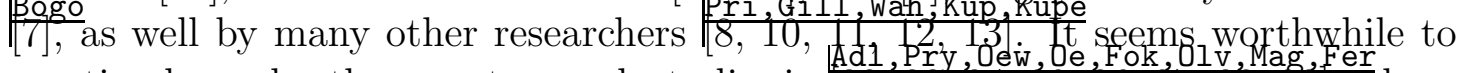
mention here also the recent enough studies in $\frac{A 22,23,24,39,26,27,28,29], \text { where }}{22,26}$ the special reduction methods were proposed for the integrable nonlinear dynamical systems on both functional and operator manifolds. In the present paper we describe in detail the reduction procedure for infinite dimensional dynamical systems upon the invariant set of critical points of some global invariant functional. 
The method uses the Cartan's differential-geometric treating of differential ideals in Grassmann algebra over the associated jet-manifold. As one of main results, we show also that both the reduced dynamical systems and their symmetries, generate the Hamiltonian flows on the invariant critical submanifolds of local and nonlocal functionals with respect to the canonical symplectic structure upon it. These results are generalized for the case of differential-difference dynamical systems being given on discrete infinite-dimensional manifolds. The direct procedure to construct the invariant Lagrangian functionals for a given apriori Lax-type integrable dynamical system is presented for both the differential and the differential-difference cases of the manifold $M$. Some remarks on the Lagrangian and Hamiltonian formalisms, concerned to infinite-dimensional dynamical systems with symmetries are given. The Hamiltonian representation for a hierarchy of Lax type equations on a dual space to the Lie algebra of integral-differential operators with matrix coefficients, extended by evolutions for eigenfunctions and adjoint eigenfunctions of the corresponding spectral problems, is obtained via some special Backlund transformation. The connection of this hierarchy with integrable by Lax spatially two-dimensional systems is studied.

\section{General setting}

We are interested in treating a given nonlinear dynamical system

$$
d u / d t=K[u]
$$

with respect to an evolution parameter $t \in \mathbb{R}$ on an infinite-dimensional functional manifold $M \subset C^{(\infty)}\left(\mathbb{R} ; \mathbb{R}^{m}\right)$, possessing two additional ingredients: a homogenous and autonomous conservation law $\mathcal{L} \in D(M)$ and a number of homogenous autonomous symmetries $d u / d t_{j}=K_{j}[u], \quad j=\overline{1, k}$, with evolution parameters $t_{j} \in \mathbb{R}$. The dynamical system $(2.1)$ is not-supposed to be in general Hamiltonian, all the maps $K, K_{j}: M \rightarrow T(M), \quad j=\overline{1, k}$, being considered smooth and well-defined on $M$.

To pose the problem to be discussed further more definitely, let us involve the jet-manifold $J^{(\infty)}\left(\mathbb{R} ; \mathbb{R}^{m}\right)$ locally isomorphic to the functional manifold $M$ mentioned above. This means the following: the vector field (2.1) on $M$ is completely equivalent to that on the jet-manifold $J^{(\infty)}\left(\mathbb{R} ; \mathbb{R}^{m}\right)$ via the representation $[1,2]$

$$
(M \ni u \rightarrow K[u]) \stackrel{j e t}{\longrightarrow}\left(\left(K\left(u, u^{(1)}, \ldots, u^{(n+1)}\right) \leftarrow\left(x ; u, u^{(1)}, \ldots, u^{(\infty)}\right) \in J^{(\infty)}\left(\mathbb{R} ; \mathbb{R}^{m}\right)\right),\right.
$$

where $n \in \mathbf{Z}_{+}$is fixed, $x \in \mathbb{R}$ is the function parameter of the jet-bundle $J^{(\infty)}\left(\mathbb{R} ; \mathbb{R}^{m}\right) \stackrel{\pi}{\longrightarrow}$ $\mathbb{R}$, and $\pi$ is the usual projection on the base $\mathbb{R}$. Let us allow also that the smooth functional $\mathcal{L} \in D(M)$ is a conservation law of the dynamical system (2.1), that is 
$d \mathcal{L} / d t=0$ along orbits of $(2.1)$ for all $t \in \mathbb{R}$. Due to the jet-representation (2.2) we can write the density of the functional $\mathcal{L} \in D(M)$ in the following form:

$$
\mathcal{L}=\int_{\mathbb{R}} d x \mathcal{L}[u]
$$

with $\mathbb{R} \times \mathbb{R}^{m} \ni[x ; u] \stackrel{\text { jet }}{\longrightarrow}\left(x ; u, u^{(1)}, \ldots, u^{(N+1)}\right) \in J^{(N+1)}\left(\mathbb{R} ; \mathbb{R}^{m}\right)$ being the standard jet-mapping and a number $N \in \mathbf{Z}_{+}$fixed. Besides, the functional (2.3) will be assumed to be non-degenerate in the sense that Hessian of $\mathcal{L}: J^{(N+1)}\left(\mathbb{R} ; \mathbb{R}^{m}\right) \rightarrow \mathbb{R}$ has nonvanishing determinant: $\operatorname{det}\left\|\frac{\partial^{2} \mathcal{L}\left(u, u^{(1)}, \ldots, u^{(N+1)}\right)}{\partial u^{(N+1)} \partial u^{(N+1)}}\right\| \neq 0$.

\section{Lagrangian reduction}

Consider now the set of critical points $M_{n} \subset M$ of the functional $\mathcal{L} \in D(M)$ :

$$
M_{N}=\{u \in M: \operatorname{grad} \mathcal{L}[u]=0\}
$$

where, due to $(2.2), \operatorname{grad} \mathcal{L}[u]_{\mathrm{tax}}=\delta \mathcal{L}\left(u, \ldots, u^{(N+1)}\right) / \delta u$ - the Euler variational derivative. As proved by Lax [3], the manifold $M_{N} \subset M$ is smoothly imbedded well-defined one due to the condition Hess $\mathcal{L} \neq 0$. Besides, the manifold $M_{N}$ appears to be invariant with respect to the dynamical system (2.1). This means in particular that the Lie-derivative of any vector field $X: M \rightarrow T(M)$, tangent to the manifold $M_{N}$ with respect to the vector field (2.1), is again tangent to $M_{N}$, that isthe followung implication

$$
X[u] \in T_{u}\left(M_{N}\right) \Rightarrow[K, X][u] \in T_{u}\left(M_{N}\right)
$$

holds for all $u \in M_{N}$. Here we are in a position to begin with a study of the intrinsic structure of the manifold $M_{N} \subset M_{\text {within }}$ the geometric Cartan's theory developed

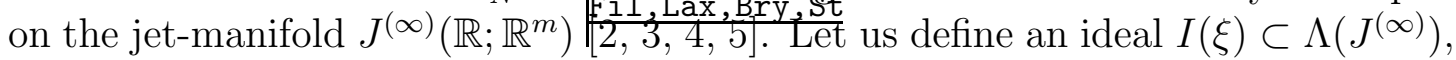
generated by the vector one-forms $\xi_{j}^{(1)}=d u^{(j)}-u^{(j+1)} d x, \quad j \in \mathbf{Z}_{+}$, which are canceled the vector field $d / d x$ on the jet-manifold $J^{(\infty)}\left(\mathbb{R} ; \mathbb{R}^{m}\right)$ :

$$
i_{\frac{d}{d x}} \xi_{j}^{(1)}=0, \quad j \in \mathbf{Z}_{+}
$$

where $x \in \mathbb{R}$ belongs to the jet-bundle base, $i_{\frac{d}{d x}}-$ the intrinsic derivative along the vector field

$$
\frac{d}{d x}=\frac{\partial}{\partial x}+\sum_{j \in \mathbf{Z}_{+}}<u^{(j+1)}, \frac{\partial}{\partial u^{(j)}}>,
$$

where $\langle.,$.$\rangle is the standard scalar product in \mathbb{R}^{m}$. The vector field (2.1) on the jet-manifold $J^{(\infty)}(\mathbb{R} ; \mathbb{R})$ has the analogous representation:

$$
\frac{d}{d t}=\frac{\partial}{\partial t}+\sum_{j=\mathbf{Z}_{+}}<K^{(j)}, \frac{\partial}{\partial u^{(j)}}>,
$$


where, by definition, $K^{(j)}:=\frac{d^{j}}{d x^{j}} K, j=\mathbf{Z}_{+}$. The problem arises: how to build the intrinsic variables on the manifold $M_{N} \subset M$ from the jet-manifold coordinates on $\left.J^{(\infty)}\left(\mathbb{R} ; \mathbb{R}^{m}\right)\right)$ ?

To proceed to the solution of the problem above, let us study the 1-form $d \mathcal{L}=$ $\Lambda^{1}\left(J^{(\infty)}(\mathbb{R} ; \mathbb{R})\right)$ as one defined on the submanifold $M_{N} \subset M$. We have the following chain of identities in the Grassmann subalgebra $\Lambda\left(J^{(2 N+2)}\left(\mathbb{R} ; \mathbb{R}^{m}\right)\right)$ :

$$
\begin{aligned}
d \mathcal{L} & =d\left(i_{\frac{d}{d x}} \mathcal{L} d x\right)=d i_{\frac{d}{d x}}\left(\mathcal{L} d x+\sum_{j=0}^{N}\left\langle p_{j}, \mathbb{R}\right\rangle\right) \\
& =\left(d i_{\frac{d}{d x}}+i_{\frac{d}{d x}} d\right) \cdot\left(\mathcal{L} d x+\sum_{j=0}^{N}\left\langle p_{j}, \xi_{j}^{(1)}\right\rangle\right)-i_{\frac{d}{d x}} d\left(\mathcal{L} d x+\sum_{j=0}^{N}\left\langle p_{j}, \xi_{j}^{(1)}\right\rangle\right),
\end{aligned}
$$

where $p_{j}: J^{(2 N+2)}\left(\mathbb{R} ; \mathbb{R}^{m}\right) \rightarrow \mathbb{R}^{m}, j=\overline{0, N}$, are some until not definite vectorfunctions. Requiring now that 2-form $d\left(\mathcal{L} d x+\sum_{j=0}^{N}\left\langle p_{j}, \xi_{j}^{(1)}\right\rangle\right)$ do not depend on differentials $d u^{(j)}, j=\overline{1, N+1}$, that is

$$
i_{\frac{\partial}{\partial u^{(j)}}}\left(d \mathcal{L} \wedge d x+\sum_{k=0}^{N}\left\langle d p_{k} \wedge \xi_{j}^{(1)}\right\rangle\right)=0,
$$

one can determine thus the vector-functions $p_{j}=\mathbb{R}^{m}, \quad j=\overline{0, N}$. As a result we obtain the following simple recurrent relations:

$$
\frac{d p_{j}}{d x}+p_{j-1}=\frac{\partial \mathcal{L}}{\partial u^{(j)}}
$$

for $j=\overline{1, N+1}$, setting $p_{-1}=0=p_{N+1}$ by definition. The unique solution to (3.7) is made by the following expressions, $j=\overline{0, N}$ :

$$
p_{j}=\sum_{k=0}^{N}(-1)^{k} \frac{d^{k}}{d x^{k}} \frac{\partial \mathcal{L}}{\partial u^{(j+k+1)}} .
$$

Thereby we have got, owing (3.5) and (3.6), the following final representation for the differential $d \mathcal{L}$ :

$$
\begin{aligned}
d \mathcal{L}= & \frac{d}{d x}\left[\mathcal{L}-\sum_{j=0}^{N}\left\langle p_{j}, u^{(j+1)}\right\rangle\right] d x-\left\langle\operatorname{grad} \mathcal{L}[u], u^{(1)}\right\rangle d x+ \\
& \left.+\frac{d}{d x}\left(\sum_{j=0}^{N}\left\langle p_{j}, d u^{(j)}\right\rangle\right]\right)+\langle\operatorname{grad} \mathcal{L}[u], d u\rangle,
\end{aligned}
$$

with $\frac{d}{d x}:=d i_{\frac{d}{d x}}+i_{\frac{d}{d x}} d$ being the Lie-derivative along the vector field $\frac{d}{d x}$, and $\operatorname{grad} \mathcal{L}[u]:=\delta \mathcal{L} / \delta u$ as it was mentioned above in the chapter 2. Below we intend to treat the representation (3.9) on the topic of a symplectic structure arisen from the above analysis on the invariant submanifold $M_{N} \subset M$. 


\section{Symplectic analysis and Hamiltonian formula- tion}

Let us put into the expression (3.9) the condition $\operatorname{grad} \mathcal{L}[u]=0$ for all $u=M_{N}$. Then the following equality is satisfied:

$$
d \mathcal{L}=\frac{d}{d x} \alpha^{(1)}, \quad \alpha^{(1)}=\sum_{j=0}^{N}\left\langle p_{j}, d u^{(j)}\right\rangle,
$$

since the function $h^{(x)}:=\sum_{j=0}^{N}\left\langle p_{j}, u^{(j+1)}\right\rangle-\mathcal{L}\left(u, \ldots, u^{(N+1)}\right)$ satisfies the condition $d h^{(x)} / d x=-\left\langle\operatorname{grad} \mathcal{L}[u], u^{(1)}\right\rangle$ for all $x=\mathbb{R}$, owing to the relations (3.7). Taking now the external derivative of (4.1), we obtain that

$$
\frac{d}{d x} \Omega^{(2)}=0, \quad \Omega^{(2)}=d \alpha^{(1)}
$$

where we have used the well known identity $d \cdot \frac{d}{d x}=\frac{d}{d x} \cdot d$. From (4.2) we can conclude that the vector field $d / d x$ on the submanifold $M_{N} \subset M$ is Hamiltonian with respect to the canonical symplectic structure $\Omega^{(2)}=\sum_{j=0}^{N}\left\langle d p_{j} \wedge d u^{(j)}\right\rangle$. It is a very simple exercise to state that the function $h^{(x)}: J^{(2 N+2)}\left(\mathbb{R} ; \mathbb{R}^{m}\right) \rightarrow \mathbb{R}$ defined above is playing a role of the corresponding Hamiltonian function for the vector field $d / d x$ on $M_{N}$, i.e. the equation

$$
d h^{(x)}=-i_{\frac{d}{d x}} \Omega^{(2)}
$$

holds on $M_{N}$. Therefore, we have got the following theorem.

Theorem 1. The critical submanifold $M_{N} \subset M$ defined by (3.1) for a given non degenerate smooth functional $L=D(M) \subset D\left(J^{(N+1)}\left(\mathbf{R} ; \mathbf{R}^{m}\right)\right)$, being imbedded into the jet-manifold $J^{(\infty)}\left(\mathbf{R} ; \mathbf{R}^{m}\right)$, carries the canonical symplectic structure subject to which the induced vector field $d / d x$ on $M_{N}$ is Hamiltonian.

The theorem analogous to the that above was stated before via different manners by many authors $\left[\frac{P r}{8}, 9\right]$. Our derivation presented here is as much simpler as constructive, giving rise to all ingredients of symplectic theory, stemming from imbedding the invariant submanifold $M_{N}$ into the jet-manifold.

Now we are going to proceed further to studying the vector field (2.1) on the manifold $M_{N} \subset M$ endowed with the symplectic structure $\Omega^{(2)}=\Lambda^{2}\left(J^{(N+1)}\left(\mathbb{R} ; \mathbb{R}^{m}\right)\right)$, having built via the formula (4.2).

We have the following implicating identities:

$$
\begin{aligned}
& \frac{d \mathcal{L}}{d t}=0 \Rightarrow \quad\langle\operatorname{grad} \mathcal{L}[u], K[u]\rangle=-\frac{d h^{(t)}}{d x}, \\
& \frac{d \mathcal{L}}{d x}=0 \Rightarrow \quad\left\langle\operatorname{grad} \mathcal{L}[u], \frac{d u}{d x}\right\rangle=-\frac{d h^{(x)}}{d x}
\end{aligned}
$$


where functions $h^{(t)}$ and $h^{(x)}$ serve as corresponding Hamiltonian ones for the vector fields $d / d t$ and $d / d x$. This means in part that the following equations hold:

$$
d h^{(x)}=-i_{\frac{d}{d x}} \Omega^{(2)}, \quad d h^{(t)}=-i_{\frac{d}{d t}} \Omega^{(2)} .
$$

To prove the above statement (4.5), we shall build the following quantities (for the vector field $d / d x$ at first):

$$
d \cdot i_{\frac{d}{d x}}\langle\operatorname{grad} \mathcal{L}[u], d u\rangle=-\frac{d}{d x}\left(d h^{(x)}\right)
$$

stemming from (4.4), and

$$
i_{\frac{d}{d x}} \cdot d\langle\operatorname{grad} \mathcal{L}[u], d u\rangle=-\frac{d}{d x}\left(i_{\frac{d}{d x}} \Omega^{(2)}\right)
$$

stemming from (3.9), where we used the preliminary used the evident identity $\left[i_{\frac{d}{d x}}, \frac{d}{d x}\right]=0$. Adding now the expression (4.6) and (4.7) entails the following one:

$$
\frac{d}{d x}\langle\operatorname{grad} \mathcal{L}[u], d u\rangle=-\frac{d}{d x}\left(d h^{(x)}+i_{\frac{d}{d x}} \Omega^{(2)}\right)
$$

for all $x=\mathbb{R}$ and $u=M$. Since $\operatorname{grad} \mathcal{L}[u]=0$ for all $u=M_{N}$, we obtain from (4.8) that the first equality in (4.5) is valid in the case of the vector field $d / d x$ reduced on $M_{N}$. The analogous procedure fits also for the vector field $d / d t$ reduced on the manifold $M_{N} \subset M$. The even difference of the procedure above stems from the condition on vector fields $d / d t$ and $d / d x$ to be commutative, $[d / d t, d / d x]=0$ what engenders the needed identity $\left[i_{\frac{d}{d t}}, \frac{d}{d x}\right] \equiv i_{\left[\frac{d}{d t}, \frac{d}{d x}\right]}=0$ as a simple consequence of the procedure considered above. There upon we have stated the validity of equations (4.5) completely. $\triangleright$

Theorem 2. Dynamical systems $d / d t$ and $d / d x$ reduced on the invariant submanifold $M_{N} \subset M(3.1)$ are Hamiltonian ones with the corresponding Hamiltonian functions built from the equations (4.4) in the unique way.

By the way, we have stated also that the Hamiltonian functions $h^{(x)}$ and $h^{(t)}$ on the submanifold $M_{N} \subset M$ are commuting with each other, that is $\left\{h^{(t)}, h^{(x)}\right\}=0$ where $\{\cdot, \cdot\}$ is the Poissin structure on $D\left(M_{N}\right)$ corresponding to the symplectic structure (4.2). This indeed follows from the equalities (4.4), since $\left\{h^{(t)}, h^{(x)}\right\}=$ $\frac{d h^{(x)}}{d t}=-\frac{d h^{(t)}}{d x} \equiv 0$ upon the manifold $M_{N} \subset M$.

\section{$5 \quad$ Symmetry invariance}

Let us consider now any vector field $K_{j}: M \rightarrow T(M), \quad j=\overline{1, k}$, being symmetry fields related with the given vector field $(2.1)$, i.e. $\left[K, K_{j}\right]=0,=\overline{1, k}$. As the 
conservation law $\mathcal{L} \in D(M)$ for the vector field (2.1) has not to be that for the vector fields $K_{j}, j=\overline{1, k}$, the submanifold $M_{N} \subset M$ has not to be invariant also with respect to these vector fields. Therefore, if a vector field $X \in T\left(M_{N}\right)$, the vector field $\left[K_{j}, X\right] \notin T\left(M_{N}\right)$ in general, if $\frac{d}{d t_{j}}, j=\overline{1, k}$, are chosen as symmetries of (2.1). Let us consider the following identity for some there existing function $\tilde{h}_{j}$ : $J^{(2 N+2)}\left(\mathbb{R} ; \mathbb{R}^{m}\right) \rightarrow \mathbb{R}, j=\overline{1, k}$, stemming from the conditions $\left[\frac{d}{d t}, \frac{d}{d t_{j}}\right]=0, j=\overline{1, k}$, on $M$ :

$$
\frac{d}{d t} i_{\frac{d}{d t_{j}}}<\operatorname{grad} \mathcal{L}[u], d u>=-\frac{d \tilde{h}_{j}[u]}{d x} .
$$

Lemma 1.The functions $\tilde{h}_{j}[u], j=\overline{1, k}$, reduced on the invariant submanifold $M_{N} \subset M$ turn into constant ones. These constants can be chosen obviously as zero ones.

Proof. We have: $\left[\frac{d}{d t}, i_{\frac{d}{d t_{j}}}\right]=0, j=\overline{1, k}$, there upon

$$
\begin{gathered}
i_{\frac{d}{d t_{j}}}\left(i_{\frac{d}{d t}} d+d i_{\frac{d}{d t}}\right)<\operatorname{grad} \mathcal{L}[u], d u>=-\frac{d \tilde{h}_{j}}{d x} \Rightarrow \\
\Rightarrow i_{\frac{d}{d t_{j}}}\left(i_{\frac{d}{d t}} d<\operatorname{grad} \mathcal{L}[u], d u>+d i_{\frac{d}{d t}}<\operatorname{grad} \mathcal{L}[u], d u>\right) \\
=-i_{\frac{d}{d t_{j}}} i_{\frac{d}{d t}} \frac{d}{d x} \Omega^{(2)}-i_{\frac{d}{d t_{j}}} \frac{d}{d x}\left(d h^{(+)}\right)=-\frac{d}{d x} i_{\frac{d}{d t_{j}}}\left(i_{\frac{d}{d t}} \Omega^{(2)}+d h^{(t)}\right)=-\frac{d \tilde{h}_{j}}{d x} .
\end{gathered}
$$

Whence we obtain that upon the whole jet-manifold $M \subset J^{\infty}\left(\mathbb{R} ; \mathbb{R}^{m}\right)$ the following identities hold:

$$
i_{\frac{d}{d t_{j}}}\left(d h^{(t)}+i_{\frac{d}{d t}} \Omega^{(2)}\right)=\tilde{h}_{j}
$$

for all $j=\overline{1, k}$. Since upon the submanifold $M_{N} \subset M$ onr has $i_{\frac{d}{d t}} \Omega^{(2)}=-d h^{(t)}$, we find that $\tilde{h}_{j} \equiv 0, j=\overline{1, k}$, that proves the lemma. $\triangleright$

Note 1 . The result above could be stated also using the standard functional operator calculus of [8]. Indeed,

$$
\begin{aligned}
& \frac{d}{d t}\left(i_{\frac{d}{d t_{j}}}<\operatorname{grad} \mathcal{L}[u], d u>\right) \\
& \left.\quad=\frac{d}{d t}<\operatorname{grad} \mathcal{L}[u], K_{j}[u]\right\rangle \\
& =\left\langle\frac{d}{d t} \operatorname{grad} \mathcal{L}[u], K_{j}[u]\right\rangle+<\operatorname{grad} \mathcal{L}[u], \frac{d}{d t} K_{j}[u]> \\
& \left.=\left\langle-K^{\prime *} \operatorname{grad} \mathcal{L}[u], K_{j}[u]\right\rangle+<\operatorname{grad} \mathcal{L}[u], K_{j}^{\prime} \cdot K[u]\right\rangle \\
& \left.=-\left\langle K^{\prime *} \operatorname{grad} \mathcal{L}[u], K_{j}[u]\right\rangle+<\operatorname{grad} \mathcal{L}[u], K^{\prime} \cdot K_{j}[u]\right\rangle \\
& =-\frac{d}{d x} \mathcal{H}\left[\operatorname{grad} \mathcal{L}[u], K_{j}[u]\right]=-\frac{d \tilde{h}_{j}[u]}{d x} .
\end{aligned}
$$


Here a bilinear form $\mathcal{H}\{\cdot \cdot\}$ is found via the usual definition of the adjoined operator $K^{\prime *}$ for a given operator $K^{\prime}: L_{2} \rightarrow L_{2}$ with respect to the natural scalar bracket $(\cdot, \cdot)$

$$
\left(K^{\prime *} a, b\right):=\left(a, K^{\prime} b\right), \quad(a, b):=\int_{\mathbb{R}} d x\langle a, b\rangle,
$$

whence we simply obtain:

$$
\left\langle K^{\prime *} a, b\right\rangle-\left\langle a, K^{\prime} b\right\rangle=d \mathcal{H}[a, b] / d x
$$

for all $a, b \in L_{2}$. Therefore, we can identify $\tilde{h}_{j}[u]=\mathcal{H}\left[\operatorname{grad} \mathcal{L}[u], K_{j}[u]\right]$ for all $u \in M, j=\overline{1, k}$. If $u \in M_{N}$, we therewith obtain that $\tilde{h}_{j}[u] \equiv 0, \quad j=\overline{1, k}$, that was needed to prove. $\triangleright$

As a result of the Lemma proven above one gets the following: the functions $\tilde{h}_{j}[u], j=\overline{1, k}$, can not serve as nontrivial Hamiltonian ones for the dynamical systems $d / d t_{j}, j=\overline{1, k}$, upon the submanifold $M_{N} \subset M$. To overcome this difficulty we assume the invariant submanifold $M_{N} \subset M$ to possess some additional symmetries $d / d t_{j}, j=\overline{1, k}$, which satisfy the following characteristic criterion: $L_{\frac{d}{d t_{j}}} \operatorname{grad} \mathcal{L}[u]=0, j=\overline{1, k}$, for all $u \in M_{N}$. This means that for $j=\overline{1, k}$

$$
L_{\frac{d}{d t_{j}}} \operatorname{grad} \mathcal{L}[u]=G_{j}(\operatorname{grad} \mathcal{L}[u])
$$

where $G_{j}(\cdot), j=\overline{1, k}$, are some linear vector-valued functionals on $T^{*}(M)$. Otherwise, equations (5.6) are equivalent to the following:

$$
\left.i_{\frac{d}{d t_{j}}}<\operatorname{grad} \mathcal{L}[u], d u\right\rangle=-d h_{j}[u] / d x+g_{j}(\operatorname{grad} \mathcal{L}[u])
$$

where $g_{j}(\cdot), j=\overline{1, k}$, are some scalar linear functionals on $T^{*}(M)$. From (3.9) and (5.7) we therewith find that for all $j=\overline{1, k}$

$$
L_{\frac{d}{d t_{j}}}\langle\operatorname{grad} \mathcal{L}[u], d u\rangle=-\frac{d}{d x}\left(d h_{j}[u]+i_{\frac{d}{d t_{j}}} \Omega^{(2)}\right)+d g_{j}(\operatorname{grad} \mathcal{L}[u]) .
$$

If we put now $u \in M_{N}$, that is $\operatorname{grad} \mathcal{L}[u]=0$, we immediately will find the following: for all $j=\overline{1, k}$,

$$
d h_{j}[u]+i_{\frac{d}{d t_{j}}} \Omega^{(2)}=0 .
$$

Whence we can make a conclusion that the vector fields $d / d t_{j}, j=\overline{1, k}$, are Hamiltonian too on the submanifold $M_{N} \subset M$. Since $d h_{j} / d x=\left\{h^{(x)}, h_{j}\right\}=0, j=\overline{1, k}$, on the manifold $M_{N}$, we therewith obtain that $d h^{(x)} / d t_{j}=0, j=\overline{1, k}$. This is also an obvious corollary of the commutativity $\left[d / d t_{j}, d / d x\right]=0, j=\overline{1, k}$, for all $x, t_{j} \in \mathbb{R}$ on the whole manifold $M$. Indeed, in general case we have the identity 
$\left\{h^{(x)}, h_{j}\right\}=i_{\left[\frac{d}{d t_{j}}, \frac{d}{d x}\right]} \Omega^{(2)}$, whence the equalities $\left\{h^{(x)}, h_{j}\right\} \equiv 0$ hold for all $j=\overline{1, k}$

on the submanifold $M_{N} \subset M$, since $\left[\frac{d}{d t_{j}}, \frac{d}{d x}\right]=0$ on $M_{N}$ due to (5.8). The analysis fulfilled above makes it possible to treat given vector fields $d / d t_{j}, j=\overline{1, k}$, satisfying either conditions (5.6) or conditions (5.7) on the canonical symplectic jet-submanifold $M_{N} \subset M$ analytically as Hamiltonian systems.

\section{Liouville integrability}

Now we suppose that the vector field $d / d t_{j}, j=\overline{1, k}$, are independent and commutative both to each other on the jet-submanifold $M_{N} \subset M$ and with vector fields $d / d t$ and $d / d x$ on the manifold $M$. Besides, the submanifold $M_{N} \subset M$ is assumed to be compact and smoothly imbedded into the jet-manifold $\left.J_{\text {ri }, G e} J^{(\infty)} ; \mathbb{R}^{m}\right)$. If the dimension $\operatorname{dim} M_{N}=2 k+4$, due to the Liouville theorem $\left.\frac{p r}{8}, 9\right]$ the dynamical systems $d / d x$ and $d / d t$ are Hamiltonian and integrable by quadratures on the submanifold $M_{N} \subset M$. This is the case for all Lax-integrable nonlinear dynamical systems of the Korteweg-de Vries type $43,7,9,8]$ on spatially one-dimensonal functional manifolds.

\section{Discrete dynamical systems}

Let us be given a differential discrete smooth dynamical system

$$
d u_{n} / d t=K_{n}[u]
$$

with respect to a continuous evolution parameter $t \in \mathbb{R}$ on the infinite-dimensional discrete manifold $M \subset L_{2}\left(\mathbf{Z} ; \mathbb{R}^{m}\right)$ infinite vector-sequences under the condition of rapid decrease in $n \in \mathbf{Z}: \sup _{n \in \mathbf{Z}}|n|^{k}\left\|u_{n}\right\|_{\mathbb{R}^{m}}<\infty$ for all $k \in \mathbf{Z}_{+}$at each point $u=\left(\ldots, u_{n}, u_{n+1}, \ldots\right) \in M$, where $u_{n} \in \mathbb{R}^{m}, \quad n \in \mathbf{Z}$.

Assume further that the dynamical system (7.1) possesses a conservation law $\mathcal{L} \in D(M)$, that is $d \mathcal{L} / d t=0$ along orbits of (7.1). Via the standard operatorial analysis one gets from (3.5) the variational derivative of a functional $\mathcal{L}:=\sum_{n \in \mathbf{Z}} \mathcal{L}_{n}[u]:$

$$
\operatorname{grad} \mathcal{L}_{n}:=\delta \mathcal{L}[u] / \delta u_{n}=\mathcal{L}_{n}^{*}[u] \cdot 1,
$$

where the last right-hand operation of multiplying by unity is to be fulfilled by component.

Lemma 2. Let $\Lambda(M)$ be the infinite-dimensional Grassmannian algebra on the manifold $M$; then the differential $d L_{n}[u] \in \Lambda^{1}(M)$ enjoys the following reduced representation:

$$
\left.d \mathcal{L}_{n}[u]=<\operatorname{grad} \mathcal{L}_{n}, d u_{n}\right\rangle+d / d n \alpha_{n}^{(1)}[u]
$$


where a one-form $\alpha_{n}^{(1)}[u] \in \Lambda^{1}(M)$ is determined in unique way, $\langle\cdot, \cdot\rangle$ is the usual scalar product in $R^{m}$ and $d / d n=\Delta-1, \quad \Delta$ is the usual shift operator.

Proof. By definition we obtain for the external differential $d \mathcal{L}_{n}[u]$ the following chain of representations for each $n \in \mathbf{Z}$ :

$$
\begin{gathered}
d \mathcal{L}_{n}[u]=\sum_{k=0}^{N}<\frac{\partial \mathcal{L}_{n}[u]}{\partial u_{n+k}}, d u_{n+k}> \\
=\sum_{k=0}^{N} \sum_{s=0}^{k} \frac{d}{d n}<\frac{\partial \mathcal{L}_{n-s}[u]}{\partial u_{n+k-s}}, d u_{n+k-s}>+\sum_{k=0}^{N}<\frac{\partial \mathcal{L}_{n-k}[u]}{\partial u_{n}}, d u_{n}> \\
=\frac{d}{d n} \sum_{k=0}^{N} \sum_{s=0}^{k}<\frac{\partial \mathcal{L}_{n-s}[u]}{\partial u_{n+k-s}}, d u_{n+k-s}>+\sum_{k=-N}^{N} \triangle^{-k}<\frac{\partial \mathcal{L}_{n}[u]}{\partial u_{n+k}}, d u_{n}> \\
=\frac{d}{d n} \sum_{k=0}^{N} \sum_{s=0}^{k}<\frac{\partial \mathcal{L}_{n-s}[u]}{\partial u_{n+k-s}}, d u_{n+k-s}>+<\mathcal{L}_{n}^{\prime *} \cdot 1, d u_{n}> \\
\left.=\frac{d}{d n} \alpha_{n}^{(1)}[u]+<\operatorname{grad} \mathcal{L}_{n}[u], d u_{n}\right\rangle,
\end{gathered}
$$

where $N \in \mathbf{Z}_{+}$is the fixed number depending on the jet-form of a functional $\mathcal{L} \in D(M)$

$$
\begin{gathered}
\alpha_{n}^{(1)}[u]=\sum_{k=0}^{N} \sum_{s=0}^{k}<\frac{\partial \mathcal{L}_{n-s}[u]}{\partial u_{n+k-s}}, d u_{n+k-s}> \\
=\sum_{k=0}^{N} \sum_{j=0}^{k}<\frac{\partial \mathcal{L}_{n+j-k}[u]}{\partial u_{n+j}}, d u_{n+j}>,
\end{gathered}
$$

and

$$
\operatorname{grad} \mathcal{L}_{n}[u]=\mathcal{L}_{n}^{\prime *} \cdot 1=\sum_{k=0}^{N} \frac{\partial \mathcal{L}_{n-k}[u]}{\partial u_{n}} .
$$

The latter equality in (7.4) proves the lemma 2 completely. $\triangleright$

The above proved representation (7.3) gives rise to the following stationary problem being posed on the manifold $M$ :

$$
M_{N}=\left\{u \in M: \operatorname{grad} \mathcal{L}_{n}=0\right\}
$$

for all $n \in \mathbf{Z}$, where by definition $\operatorname{det}\left\|\frac{\partial^{2} \mathcal{L} n[u]}{\partial u_{N+1} \partial u_{N+1}}\right\|=0$. In virtue of (7.3) we obtain the validity of the following theorem. 
Theorem 3. The finite-dimensional Lagrangian submanifold $M_{N} \subset M$ defined by (7.6), is a symplectic one with the canonical symplectic structure $\Omega_{n}^{(2)}=d \alpha_{n}^{(1)}$ being independent of the discrete variable $n \in \mathbf{Z}$.

Proof. From (7.3) we have that on the manifold $M_{N} \subset M \quad d \mathcal{L}_{n}[u]=d / d n\left(\alpha_{n}^{(1)}[u]\right)$, whence for all $n \in \mathbf{Z} \quad d / d n\left(\Omega_{n}^{(2)}\right)=0$. This means obviously, that $\Omega_{n+1}^{(2)}=\Omega_{n}^{(2)}$ for all $n \in \mathbf{Z}$, or equivalently, the 2 -form $\Omega_{n}^{(2)}$ is not depending on the discrete variable $n \in \mathbf{Z}$. As the 2 -form $\Omega_{n}^{(2)}:=d \alpha_{n}^{(1)}$ by definition, this form is chosen to be a symplectic form on the manifold $M_{N} \subset M$. For this 2-form to be nondegenerate on $M_{N}$, we assume that Hessian of $\mathcal{L}_{n}$ equals $\operatorname{det}\left\|\frac{\partial^{2} \mathcal{L}_{n}[u]}{\partial u_{n+N+1} \partial u_{n+N+1}}\right\| \neq 0$ on $M_{N}$. The latter proves the theorem. $\triangleright$

Let us consider now the prior given dynamical system (7.1) reduced on the submanifold $M_{N} \subset M$. To present it as the vector field $d / d t$ on $M_{N}$, we need preliminary to represent it as a Hamiltonian flow on $M_{N}$. To do this, let us write the following identities on $M$ :

$$
\begin{aligned}
& \left.i_{\frac{d}{d t}} d<\operatorname{grad} \mathcal{L}_{n}, d u_{n}\right\rangle=-\frac{d}{d n} i_{\frac{d}{d t}} \Omega_{n}^{(2)}[u], \\
& \left.d i_{\frac{d}{d t}}<\operatorname{grad} \mathcal{L}_{n}, d u_{n}\right\rangle=-\frac{d}{d n}\left(d h_{n}^{(t)}[u]\right),
\end{aligned}
$$

which are valid for all $n \in \mathbf{Z}$. Adding the last identities in (7.7), we come to the following one for all $n \in \mathbf{Z}$ :

$$
\left.\frac{d}{d t}<\operatorname{grad} \mathcal{L}_{n}, d u_{n}\right\rangle=-\frac{d}{d n}\left(i_{\frac{d}{d t}} \Omega_{n}^{(2)}[u]+h_{n}^{(t)}[u]\right) .
$$

Having reduced the identity (7.8) upon the manifold $M_{N} \subset M$, we obtain the wanted expression for all $u \in M_{N}, \quad N \in \mathbf{Z}$ :

$$
i_{\frac{d}{d t}} \Omega_{n}^{(2)}[u]+h_{n}^{(t)}[u]=0 .
$$

The latter means that the dynamical system (7.1) on the manifold $M_{N}$ is a Hamiltonian one, with the function $h_{n}^{(t)}[u]$ being a Hamiltonian one defined explicitly by the second identity in (7.7).

We assume now that the symplectic structure $\Omega_{n}^{(2)}[u]$ on $M_{N}$ be representable as follows:

$$
\Omega_{n}^{(2)}[u]=\sum_{j=0}^{N}\left\langle d p_{j+n} \wedge d u_{j+n}\right\rangle,
$$

where generalized coordinates $p_{j+n} \in \mathbb{R}^{m}, \quad j=\overline{0, N}$, are determined from the 
following discrete jet-expression $\mathcal{L}_{n}[u]:=\mathcal{L}\left(u_{n}, u_{n+1}, \ldots, u_{n+N+1}\right), \quad n \in \mathbf{Z}$,

$$
\begin{aligned}
\alpha_{n}^{(1)}[u]:=\sum_{j=0}^{N}\left\langle p_{j+n}, d u_{j+n}\right\rangle & =\sum_{k=0}^{N} \sum_{j=0}^{k}<\frac{\partial \mathcal{L}_{n+j-k}}{\partial u_{n+j}}, d u_{n+j}> \\
& =\sum_{j=0}^{N} \sum_{k=j}^{N}<\frac{\partial \mathcal{L}_{n+j-k}}{\partial u_{n+j}}, d u_{n+j}>,
\end{aligned}
$$

whence we get the final expression:

$$
p_{j+n}:=\sum_{k=j}^{N} \frac{\partial \mathcal{L}_{n+j-k}[u]}{\partial u_{n+j}},
$$

where $j=\overline{0, N}, \quad u \in M_{N} \subset M$.

Now we are in a position to reformulate the given dynamical system (7.1) as that on the reduced manifold $M_{N} \subset M$ :

$$
\frac{d u_{n+j}}{d t}=\left\{h_{n}^{(t)}, u_{n+j}\right\}=\frac{\partial h_{n}^{(t)}}{\partial p_{n+j}}, \quad \frac{d p_{n+j}}{d t}=\left\{h_{n}^{(t)}, p_{n+j}\right\}=-\frac{\partial h_{n}^{(t)}}{\partial u_{n+j}}
$$

for all $n \in \mathbf{Z}, j=\overline{0, N}$. Thereby the problem of embedding a given discrete dynamical system (7.1) into the vector field flow on the manifold $M_{N} \subset M$ is solved completely with the final result (7.12).

\section{Invariant Lagrangians construction: functional manifold case}

In the case the given nonlinear dynamical system (2.1) being integrable one of Lax-type, we can proceed effectively to find a commuting infinite hierarchy of conservation laws can be serving as the invariant Lagrangians, having been under consideration above.

At first we have to use the important property $\frac{\mathbb{F a x}}{3]}$ of the complexified gradient functional $\varphi=\operatorname{grad} \gamma \in T^{*}(M) \otimes \mathbb{C}$ generated by an arbitrary conservation law $\gamma \in D(M)$, i.e. the following Lax-type equation :

$$
d \varphi / d t+K^{*} \varphi=0
$$

where the prime sign denotes the usual Frechet derivative of the local functional $K$ : $M \rightarrow T(M)$ on the manifold $M$, the star "*" denotes its conjugation operator with respect to the nondegenerate standard convolution functional $(\cdot, \cdot)=\int_{\mathbb{R}} d x\langle\cdot, \cdot\rangle$ on 
$T^{*}(M) \times T(M)$. The equation (8.1) admits (what follows from $\frac{\text { Mit, Nov, Prjk }}{30,31,32] \text { ) }}$ the special asymptotic solution:

$$
\varphi(x, t ; \lambda) \cong(1, a(x, t ; \lambda))^{\tau} \exp \left[\omega(x, t ; \lambda)+\partial^{-1} \sigma(x, t ; \lambda)\right],
$$

where $a(x, t ; \lambda) \in \mathbb{C}^{m-1}, \quad \sigma(x, t ; \lambda) \in \mathbb{C}, \quad \omega(x, t ; \lambda)$ - some dispersive function. The sign " $\tau$ " means here the transposition one, what is adopted in matrix analysis. For any complex parameter $\lambda \in \mathbb{C}$ at $|\lambda| \rightarrow \infty$ the following expansions take place:

$$
a(x, t ; \lambda) \simeq \sum_{j \in \mathbf{Z}_{+}} a_{j}[x, t ; u] \lambda^{-j+s(a)}, \sigma(x, t ; \lambda) \simeq \sum_{j \in \mathbf{Z}_{+}} \sigma_{j}[x, t ; u] \lambda^{-j+s(\sigma)} .
$$

Here $s(a)$ and $s(\sigma) \in \mathbf{Z}_{+}$- some appropriate nonnegative integers, the operation $\partial^{-1}$ means the inverse one to the differentiation $d / d x$, that is $d / d x \cdot \partial^{-1}=1$ for all $x \in \mathbb{R}$.

To find the explicit form of the representation (8.2) in the case when the associated Lax-type representation $\frac{1 \mathrm{r} 1}{8]}$ depends parametrically on the spectral parameter $\lambda(t ; z) \in \mathbb{C}$, satisfying the following non-isospectral condition:

$$
d \lambda(t ; z) / d t=g(t ; \lambda(t ; z)),\left.\quad \lambda(t ; z)\right|_{t=0^{+}}=z \in \mathbb{C},
$$

for some meromorphic function $g(t ; \cdot): \mathbb{C} \rightarrow \mathbb{C}, t \in \mathbb{R}_{+}$, we must reanalyze more carefully the asymptotic solutions to the Lax equation (8.1). Namely, we are going to treat more exactly the case when the solution $\varphi \in T^{*}(M)$ to (8.1) is represented as an appropriate trace-functional of a Lax spectral problem at the moment $\tau=$ $t \in \mathbb{R}_{+}$with the spectral parameter $\lambda(t ; \lambda) \in \mathbb{C}$ satisfying the condition (8.3), the evolution of the given dynamical system (2.1) being considered with respect to the introduced above parameter $\tau \in \mathbb{R}$, that is

$$
d u / d \tau=K[x, \tau ; u]
$$

$\left.u\right|_{\tau=0}=\bar{u} \in M-$ some Cauchy data on $M$. This means that the functional

$$
\tilde{\varphi}(x, \tau ; \tilde{\lambda}):=\operatorname{reg} \operatorname{grad} \operatorname{Sp} S(x, \tau ; \tilde{\lambda}), \quad \tilde{\lambda}=\tilde{\lambda}(\tau ; \lambda(t ; z)) \in \mathbb{C},
$$

where $S(x, \tau ; \tilde{\lambda})$ is the monodrony matrix corresponding to a Lax type spectral problem assumed to exist, has to satisfy the corresponding Lax equation at any point $u \in M$ subject to $(8.4)$ :

$$
d \tilde{\varphi} / d \tau+K^{\prime *}[u] \cdot \tilde{\varphi}=0
$$

for all $\tau \in \mathbb{R}_{+}$. Under the above assumption it is obvious that the spectral parameter $\tilde{\lambda}=\tilde{\lambda}(\tau ; \lambda(t ; z))$, where

$$
d \tilde{\lambda} / d \tau=\tilde{g}(\tau ; \tilde{\lambda}),\left.\quad \tilde{\lambda}\right|_{\tau=0}=\lambda(t ; z) \in \mathbb{C},
$$


$\tilde{g}(t ; \cdot): \mathbb{C} \rightarrow \mathbb{C}$ - some meromorphic function being found simply from (8.6) for instance at $u=0$, the Cauchy data $\lambda(t ; z) \in \mathbb{C}$ for all $t \in \mathbb{R}_{+}$are the corresponding ones to (8.3), the parameter $z \in \mathbb{C}$ being a spectrum value of the associate Lax type spectral problem at a moment $t \in \mathbb{R}_{+}$.

Now we are in a position to formulate the following lemma.

Lemma 3. The Lax equation (8.6) as the parameter $\tau=t \in \mathbb{R}_{+}$admits an asymptotic solution in the form

$$
\tilde{\varphi}(x, \tau ; \tilde{\lambda}) \cong(1, \tilde{a}(x, \tau ; \tilde{\lambda}))^{\tau} \exp \left[\tilde{\omega}(x, \tau ; \tilde{\lambda})+\partial^{-1} \tilde{\sigma}(x, \tau ; \tilde{\lambda})\right],
$$

where $\tilde{a}(x, \tau ; \tilde{\lambda}) \in \mathbb{C}^{m-1}, \quad \tilde{\sigma}(x, \tau ; \tilde{\lambda}) \in \mathbb{C}$, are some local functionals on $M, \tilde{\omega}(x, \tau ; \tilde{\lambda}) \in$ $\mathbb{C}$ is some dispersion function for all $x \in \mathbb{R}, \tau \in \mathbb{R}_{+}$, and if for $|\lambda| \rightarrow \infty$ the property $|\tilde{\lambda}| \rightarrow \infty$ as $\tau=t \in \mathbb{R}_{+}$holds, the following expansions follows:

$$
\tilde{a}(x, \tau ; \tilde{\lambda}) \simeq \sum_{j \in \mathbf{Z}_{+}} \tilde{a}_{j}[x, \tau ; u] \tilde{\lambda}^{-j+s(\tilde{a})}, \tilde{\sigma}(x, \tau ; \tilde{\lambda}) \simeq \sum_{j \in \mathbf{Z}_{+}} \tilde{\sigma}_{j}[x, \tau ; u] \tilde{\lambda}^{-j+s(\tilde{\sigma})}
$$

with $s(\tilde{a})$ and $s(\tilde{\sigma}) \in \mathbf{Z}_{+}$being some integers.

Proof. In virtue of the theory of asymptotic expansions for arbitrary differential spectral problems, the result (8.8) will hold provided the representation (8.5) is valid and the spectral parameter $\lambda(t ; z) \in \mathbb{C}$ is taken subject to (8.7). But the above is the case because of the Lax-type integrability of the dynamical system (8.4). Further, due to the mentioned above integrability of (8.4) as well as due to the well known Stokes property of asymptotic solutions to linear equations like (8.1), the condition (8.3) holds for some meromorphic function $g(t ; \cdot): \mathbb{C} \rightarrow \mathbb{C}$, $t \in \mathbb{R}_{+}$, enjoing the determining property $\frac{d}{d t} \int_{\mathbb{R}} \tilde{\sigma}(x, t ; \tilde{\lambda}(t ; \lambda(t ; z))) d x=0$ for all $t \in \mathbb{R}_{+}$. The latter proves the lemma completely. $\triangleright$

As a result of Lemma 3 one ca $\mathrm{n}$ formulate the following important theorem.

Theorem 4. The Lax integrable parametrically isospectral dynamical system (8.4) as $\tau=t \in \mathbb{R}_{+}$admits an infinite hierarchy of conservation laws, in general nonuniform ones with respect to the variables $x \in \mathbb{R}, \tau \in \mathbb{R}_{+}$, which can be represented in an exact form in virtue of the asymptotic expansion (8.8) and (8.9).

Proof. Indeed, due to the expansion (8.8), we can obtain right away that the functional

$$
\tilde{\gamma}(t ; \lambda(t ; z))=\int_{\mathbb{R}} d x \tilde{\sigma}(x, t ; \tilde{\lambda}(t ; \lambda(t ; z)))
$$

does not depend at $\tau=t \in \mathbb{R}_{+}$on the parameter $t \in \mathbb{R}_{+}$, that is

$$
d \tilde{\gamma} /\left.d \tau\right|_{\tau=t \in \mathbb{R}_{+}}=0
$$

for all $t \in \mathbb{R}_{+}$. If we put also the parameter $\tau \in \mathbb{R}_{+}$to tend to $t \in \mathbb{R}_{+}$, due to $(8.5)$ we obtain that $\left.\tilde{\varphi}(x, \tau ; \tilde{\lambda})\right|_{\tau=t \in \mathbb{R}_{+}} \rightarrow \varphi(x, t ; \lambda)$ for all $x \in \mathbb{R}, t \in \mathbb{R}_{+}$and 
$\lambda(t ; z) \in \mathbb{C}$. This means that a complexified local functional $\varphi(x, t ; z) \in T^{*}(M) \otimes \mathbb{C}$ satisfies the equation (8.1) at each point $u \in M$. As an obvious result, the following identifications hold:

$$
\left.\tilde{\omega}(x, \tau ; \tilde{\lambda})\right|_{\tau=t \in \mathbb{R}_{+}} \rightarrow \omega(x, t ; z),\left.\quad \tilde{\sigma}(x, \tau ; \tilde{\lambda})\right|_{\tau=t \in \mathbb{R}_{+}} \rightarrow \sigma(x, t ; z)
$$

for all $z \in \mathbb{C}$. Hence, the functional $\gamma(z):=\left.\tilde{\gamma}(\tau ; \lambda(t ; z))\right|_{\tau=t \in \mathbb{R}_{+}}=\int_{\mathbb{R}} d x \sigma(x, t ; z) \in D(M)$ doesn't depend on the evolution parameter $t \in \mathbb{R}_{+}$and due to equation (8.1) is a conserved quantity for the nonlinear dynamical system (2.1) under consideration, i.e.

$$
d \gamma(t ; z) / d t=0
$$

for all $t \in \mathbb{R}_{+}$and $z^{6} \in \mathbb{C}$. Therefore, it makes it possible to use the equation (8.12) jointly with (8.7) for the asymptotic expansions (8.9) and (8.3) to be found in exact form. To do this we at first need to insert the asymptotic expansion (8.8) in the determining equation (8.6) for the asymptotic expansions (8.9) to be found explicitly at the moment $\tau=t \in \mathbb{R}_{+}$. Keeping in mind that at $\tau=t \in \mathbb{R}_{+} \quad|\lambda| \rightarrow$ $\infty$ if $|\tilde{\lambda}| \rightarrow \infty$, and solving step by step the resulting recurrence relationships for the coefficients in (8.9), we will get the functional $\gamma(z):=\left.\tilde{\gamma}(\tau ; \lambda(t ; z))\right|_{\tau=t \in \mathbb{R}_{+}}, \quad z \in \mathbb{C}$, in the form fitting for the criteria equation (8.12) could be used. As the second step, we need to use the differential equation (8.7) for the criteria equation (8.12) to be satisfied point-wise for all $t \in \mathbb{R}_{+}$. This means, in particular, that

$$
\begin{gathered}
\frac{d \gamma(z)}{d t}=\frac{d}{d t}\left(\left.\sum_{j \in \mathbf{Z}_{+}} \int_{\mathbb{R}} d x \tilde{\sigma}_{j}[x, \tau ; u] \tilde{\lambda}^{-j+s(\tilde{\sigma})}\right|_{\tau=t \in \mathbb{R}_{+}}\right) \\
=\left.\int_{\mathbb{R}} d x \sum_{j \in \mathbf{Z}_{+}}\left[\frac{d \tilde{\sigma}_{j}[x, \tau ; u]}{d t} \tilde{\lambda}^{-j+s(\tilde{\sigma})}+\tilde{\sigma}_{j}[x, \tau ; u] \tilde{\lambda}^{-j+s(\tilde{\sigma})-1}(s(\tilde{\sigma})-j) \frac{d \tilde{\lambda}}{d t}\right]\right|_{\tau=t \in \mathbb{R}_{+}} \Rightarrow \\
\Rightarrow \int_{\mathbb{R}} d x \sum_{j \in \mathbf{Z}_{+}}\left[\left(d \tilde{\sigma}_{j} / d t\right) \tilde{\lambda}^{-j+s(\tilde{\sigma})}+\left.\sum_{k>>-\infty}(s(\tilde{\sigma})-k) \tilde{\sigma}_{k} \tilde{g}_{j-k-1}(t) \tilde{\lambda}^{-j+s(\tilde{\sigma})}\right|_{\tau=t \in \mathbb{R}_{+}}+\right. \\
\left.+\left.\sum_{j \in \mathbf{Z}_{+}} \tilde{\sigma}_{j}[x, t ; u] \tilde{\lambda}^{-j+s(\tilde{\sigma})-1}(s(\tilde{\sigma})-j) \frac{\partial \tilde{\lambda}}{\partial \lambda} g(t ; \lambda)\right|_{\tau=t \in \mathbb{R}_{+}}\right] \equiv 0,
\end{gathered}
$$

where we have put by definition $\tilde{g}(\tau ; \tilde{\lambda}): \simeq \sum_{k>>-\infty} \tilde{g}_{k}(\tau) \tilde{\lambda}^{-k}$ for $\tau \in \mathbb{R}_{+}$and $|\tilde{\lambda}| \rightarrow \infty$. Since the spectral parameter $\lambda=\lambda(t ; z)$ at the moment $t=0^{+}$coincides 
with an arbitrary complex value $z \in \mathbb{C}$, the condition $|z| \rightarrow \infty$ together with (8.13) at the moment $t=0^{+}$gives rise to the following recurrent relationships:

$$
\begin{gathered}
\left.\sum_{j \in \mathbf{Z}_{+}}\left[\partial \tilde{\sigma}_{j} / d t+\tilde{\sigma}_{j}^{\prime} \cdot K[t ; u]+\sum_{k>>-\infty}(s(\tilde{\sigma})-k) \tilde{\sigma}_{k} \cdot \tilde{g}_{j-k-1}\right] \tilde{\lambda}^{-j+s(\tilde{\sigma})}\right|_{\tau=t \in \mathbb{R}_{+}}= \\
=\left.\sum_{j \in \mathbf{Z}_{+}} \tilde{\sigma}_{j}(s(\tilde{\sigma})-j) \frac{\partial \tilde{\lambda}}{\partial \lambda} g(t ; \lambda) \tilde{\lambda}^{-j+s(\tilde{\sigma})-1}\right|_{\tau=t \in \mathbb{R}_{+}} \equiv 0(\bmod d / d x)
\end{gathered}
$$

for all $j \in \mathbf{Z}_{+}, x \in \mathbb{R}, t \in \mathbb{R}_{+}$and $u \in M$. Having solved the algebraic relationships (8.14) for the prior unknown function $g(t ; \lambda), t \in \mathbb{R}_{+}$, we will obtain the generating functional $\gamma(z), z \in \mathbb{C}$, of conservation laws for (2.1) in exact form. This completes the constructive part of the proof of the theorem above.

From the practical point of view we need first to get the differential equation (8.7) in exact, maybe in asymptotic form and find further the dispersive function $\tilde{\omega}(x, t ; \tilde{\lambda})$ and the local generating functional $\tilde{\sigma}(x, \tau ; \tilde{\lambda})$ defined via $(8.8)$ and $(8.9)$ for all $x \in \mathbb{R}, \tau \in \mathbb{R}_{+}$and $|\tilde{\lambda}| \rightarrow \infty$, and next one can find the equation (8.3) due to the algorithm based on the relationships (8.14). This together with the possibility of applying the general scheme of the gradient-holonomic algorithm [40] gives rise to determining in many cases the above mentioned Lax-type representation completely in exact form, that successfully solves the pretty complex direct problem of the integrability theory of nonlinear dynamical systems on functional manifolds.

Having obtained the generation function $\gamma(z) \in D(M), z \in \mathbb{C}$, of an infinite hierarchy of conservation laws of the dynamical system (2.1) on the manifold $M$, we can build appropriately a general Lagrangian functional $\mathcal{L}_{N} \in D(M)$ as follows:

$$
\mathcal{L}_{N}=-\gamma_{N+1}+\sum_{j=0}^{N} c_{j} \gamma_{j}
$$

where, by definition, $\gamma(z)=\int_{\mathbb{R}} d x \sigma(x, t ; z)$ and for $|z| \rightarrow \infty$ functionals $\gamma_{j}=$ $\int_{\mathbb{R}} d x \sigma_{j}[x, t ; z], j \in \mathbf{Z}_{+}$, are conservation laws due to expansion (8.2), with $c_{j} \in$ $\mathbb{R}, j=\overline{0, N}$, being some arbitrary constants and $N \in \mathbf{Z}_{+}$being an arbitrary but fixed nonnegative integer. If the differential order of the functional $\gamma_{N+1} \in$ $D(M)$ has the highest one of the orders of functionals $\gamma_{j} \in D(M), j=\overline{0, N}$, and additionally, this Lagrangian is not degenerate, that is $\operatorname{det}\left(\operatorname{Hess}_{N+1}\right) \neq 0$, we can apply in general amost all the theory developed before, to prove that the critical submanifold $M_{N}=\left\{u \in M: \operatorname{grad} \mathcal{L}_{N}=0\right\}$ is a finite-dimensional invariant manifold inserted into the standard jet-manifold $J^{(\infty)}\left(\mathbb{R} ; \mathbb{R}^{m}\right)$ with the canonical symplectic structure subject to which our dynamical system is a finite-dimensional Hamiltonian flow on the invariant submanifold $M_{N}$. 


\section{Invariant Lagrangian construction : discrete manifold case}

Let us consider the discrete Lax integrable dynamical system on the discrete manifold $M$ without an a priory given Lax-type representation. The problem arises how to get the corresponding conservation laws via the gradient-holonomic algorithm 8. To realize this way let us study solutions to the Lax equation:

$$
d \varphi_{n} / d t+K_{n}^{\prime}[\tau, u] \cdot \varphi_{n}=0
$$

local functionals $\varphi_{n}[u] \in T_{u_{n}}^{*}(M)$ at the point $u_{n} \in M, n \in \mathbf{Z}$. In analogy to the approach of Chapter 7 we assert that equation (9.1) admits a comlexified generating solution $\varphi_{n}=\varphi_{n}(t ; \lambda) \in T_{u_{n}}^{*}(M) \otimes \mathbb{C}, n \in \mathbf{Z}$, with $z \in \mathbb{C}$ being a complex parameter in the following form:

$$
\varphi_{n}(t ; z) \cong\left(1, a_{n}(t ; z)\right)^{\tau} \exp [\omega(t ; z)]\left(\prod_{j=-\infty}^{n} \sigma_{j}(t ; z)\right)
$$

where $\omega(t ; z)$ is some dispersive function for $t \in \mathbb{R}_{+}, \quad a_{n}(t ; z) \in \mathbb{C}^{m-1}$, $\sigma_{n}(t ; z) \in \mathbb{R}$ are local functionals on $M$, having the following asymptotic expansions at $|z| \rightarrow \infty$ :

$$
a_{n}(t ; z) \simeq \sum_{j \in \mathbf{Z}_{+}} a_{n}[t ; u] z^{-j+s(a)}, \quad \sigma_{n}^{(j)}(t ; z) \simeq \sum_{j \in \mathbf{Z}_{+}} \sigma_{n}[t ; u] z^{-j+s(\sigma)}
$$

To find the explicit form of the asymptotic representation (9.2) we need to study additionally the asymptotic solutions to the following attached Lax equation with respect to the new evolution parameter $\tau \in \mathbb{R}_{+}$:

$$
d \tilde{\varphi}_{n} / d \tau+K_{n}^{\prime *}[\tau, u] \cdot \tilde{\varphi}_{n}=0,
$$

where $\tilde{\varphi}_{n} \in T_{u_{n}}^{*}(M) \otimes \mathbb{C}$, and a point $u \in M$ evolves subject to the following dynamical system:

$$
d u_{n} / d \tau=K_{n}[\tau ; u]
$$

for all $n \in \mathbf{Z}$. Having made the assumption above we can assert based on the general theory of asymptotic solutions to linear equations like (9.4), that it admits also in general another asymptotic solution in the similar form:

$$
\tilde{\varphi}_{n}(\tau ; \tilde{\lambda}) \cong\left(1, \tilde{a}_{n}(\tau ; \tilde{\lambda})\right)^{\tau} \exp [\tilde{\omega}(\tau ; \tilde{\lambda})] \prod_{j=-\infty}^{n} \tilde{\sigma}_{j}(\tau ; \tilde{\lambda})
$$


where for all $n \in \mathbf{Z}$ and at $\tau \in \mathbb{R}_{+}$the asymptotic expansions

$$
\begin{aligned}
& \tilde{a}_{n}(\tau ; \tilde{\lambda}) \simeq \sum_{j \in \mathbf{Z}_{+}} \tilde{a}_{n}^{(j)}[x, \tau ; u] \tilde{\lambda}^{-j+s(\tilde{a})} \\
& \tilde{\sigma}_{n}(\tau ; \tilde{\lambda}) \simeq \sum_{j \in \mathbf{Z}_{+}} \tilde{\sigma}_{n}^{(j)}[\tau ; u] \tilde{\lambda}^{-j+s(\tilde{\sigma})}
\end{aligned}
$$

hold. The expansions above are valid if $|\tilde{\lambda}| \rightarrow \infty$ as $|\lambda(t ; z)| \rightarrow \infty, z \in \mathbb{C}$. The latter is the case because of the Lax-integrability of the dynamical system (9.5). The evolution

$$
d \tilde{\lambda} / d \tau=\tilde{g}(\tau ; \tilde{\lambda}),\left.\quad \tilde{\lambda}\right|_{t=0}=\lambda(t ; z) \in \mathbb{C},
$$

where $\tilde{g}(\tau ; \cdot): \mathbb{C} \rightarrow \mathbb{C}$ is some meromorphic mapping for all $\tau \in \mathbb{R}_{+}$, is in general found making use of the corresponding solution to (9.4) at $u=0$.

Substituting the expansions (9.6) and (9.7) into (9.4), we obtain some recurrence relationships, giving rise to a possibility the expressions for local functionals $\tilde{\sigma}_{j}\left[t ; u_{n}\right], j \in \mathbf{Z}_{+}$, which can be found exactly. Having this done successfully, we assert that the functional

$$
\gamma(t ; z)=\left.\sum_{n \in \mathbf{Z}} \ln \tilde{\sigma}_{n}(\tau ; \tilde{\lambda})\right|_{\tau=t \in \mathbb{R}_{+}} \Rightarrow \sum_{n \in \mathbf{Z}} \ln \sigma_{n}(t ; z),
$$

where $\tilde{\lambda}=\tilde{\lambda}(\tau ; \lambda), \tau \in \mathbb{R}_{+}$, and $\lambda(t ; z) \in \mathbb{C}$, is a meromorphic solution to equation

$$
d \lambda / d t=g(t ; \lambda),\left.\lambda\right|_{t=0^{+}}=z \in \mathbb{C}
$$

with still independent meromorphic function $g(t ; \cdot)$ for almost all $t \in \mathbb{R}_{+}$. The latter can be found making use of the following determining condition: the local functional $\left.\tilde{\varphi}_{n}(\tau ; \tilde{\lambda})\right|_{\tau=t \in \mathbb{R}_{+}} \rightarrow \varphi_{n}(t ; z) \in T^{*}(M) \otimes \mathbb{C}$ for all $t \in \mathbb{R}_{+}$and $z \in \mathbb{C}$. Hence, the following equality holds immediately:

$$
\begin{gathered}
\frac{d}{d t}\left(\left.\sum_{n \in \mathbf{Z}_{+}} \ln \tilde{\sigma}_{n}(\tau ; \tilde{\lambda})\right|_{\tau=t \in \mathbb{R}_{+}}\right)= \\
\sum_{n \in \mathbf{Z}_{+}} \tilde{\sigma}_{n}^{-1}(t ; \tilde{\lambda})\left[\frac{\partial \tilde{\sigma}_{n}}{\partial t}+\tilde{\sigma}_{n}^{\prime} \cdot K_{n}[u]+\frac{\partial \tilde{\sigma}_{n}}{\partial \tilde{\lambda}} g(t ; \tilde{\lambda})+\left.\right|_{\tau=t \in \mathbb{R}_{+}}\right. \\
\left.+\left.\frac{\partial \tilde{\sigma}_{n}}{\partial \tilde{\lambda}} \frac{\partial \tilde{\lambda}}{\partial \lambda} g(t ; \lambda)\right|_{\tau=t \in \mathbb{R}_{+}}\right]=0
\end{gathered}
$$

for all $t \in \mathbb{R}_{+}$. Equating coefficients of (9.11) at all powers of the spectral parameter $\lambda(t ; z) \in \mathbb{C}$ to zero modulus $d / d n, n \in \mathbf{Z}$, we will find the recurrent relationships 
for the function $g(t ; \lambda)$ of $(9.8)$ to be determined successfully. Thereby, using the equation (9.10) and an expansion $\sigma(t ; z) \simeq \sum_{j \in \mathbf{Z}_{+}} \sigma_{j}\left[t ; u_{n}\right] z^{-j+s(\gamma)}$ for $|z| \rightarrow \infty$, where $s(\sigma) \in \mathbf{Z}_{+}$is some integer number, we obtain an infinite hierarchy of discretewise conservation laws of the initially given nonlinear dynamical system (2.1) on the manifold $M$. But because of the parametric dependence of the conservation laws built above on the evolution parameter $t \in \mathbb{R}_{+}$, we cannot use right now the theory developed before to prove the Hamiltonian properties of the corresponding vector fields on the invariant submanifolds. To do this in an appropriate way, it is necessary to augment the theory developed before in some important details.

\section{The reduction procedure on nonlocal Lagrangian submanifolds}

1. The general algebraic scheme. Let $\tilde{\mathcal{G}}:=C^{\infty}\left(\mathbb{S}^{1} ; \mathcal{G}\right)$ be a Lie algebra of loops, taking values in a matrix Lie algebra $\mathcal{G}$. By means of $\tilde{\mathcal{G}}$ one constructs the Lie algebra $\hat{\mathcal{G}}$ of matrix integral-differential operators [36]:

$$
\hat{a}:=\sum_{j \ll \infty} a_{j} \xi^{j}
$$

where the symbol $\xi:=\partial / \partial x$ signs the differentiation with respect to the independent variable $x \in \mathbb{R} / 2 \pi \mathbf{Z} \simeq \mathbb{S}^{1}$. The usual Lie commutator on $\hat{\mathcal{G}}$ is defined as:

$$
[\hat{a}, \hat{b}]:=\hat{a} \circ \hat{b}-\hat{b} \circ \hat{a}
$$

for all $\hat{a}, \hat{b} \in \hat{\mathcal{G}}$, where "o" is the product of integral-differential operators, taking the form:

$$
\hat{a} \circ \hat{b}:=\sum_{\alpha \in \mathbf{Z}_{+}} \frac{1}{\alpha !} \frac{\partial^{\alpha} \hat{a}}{\partial \xi^{\alpha}} \frac{\partial^{\alpha} \hat{b}}{\partial x^{\alpha}} .
$$

On the Lie algebra $\hat{\mathcal{G}}$ there exists the adinvariant nondegenerate symmetric bilinear form:

$$
(\hat{a}, \hat{b}):=\int_{0}^{2 \pi} \operatorname{Tr}(\hat{a} \circ \hat{b}) d x
$$

where $\operatorname{Tr}$-operation for all $\hat{a} \in \hat{\mathcal{G}}$ is given by the expression:

$$
\operatorname{Tr} \hat{a}:=\operatorname{res}_{\xi} \operatorname{Sp} \hat{a}=\operatorname{Sp} a_{-1},
$$

with $S p$ being the usual matrix trace. With the scalar product (10.4) the Lie algebra $\hat{\mathcal{G}}$ is transformed into a metrizable one. As a consequence, the dual to $\hat{\mathcal{G}}$ 
linear space of the matrix integral-differential operators $\hat{\mathcal{G}}^{*}$ is naturally identified with the Lie algebra $\hat{\mathcal{G}}$, that is $\hat{\mathcal{G}}^{*} \simeq \hat{\mathcal{G}}$.

The linear subspaces $\hat{\mathcal{G}}_{+} \subset \hat{\mathcal{G}}$ and $\hat{\mathcal{G}}_{-} \subset \hat{\mathcal{G}}$ such as

$$
\begin{aligned}
& \hat{\mathcal{G}}_{+}:=\left\{\hat{a}:=\sum_{j=0}^{n(\hat{a}) \ll \infty} a_{j} \xi^{j}: a_{j} \in \tilde{\mathcal{G}}, j=\overline{0, n(\hat{a})}\right\}, \\
& \hat{\mathcal{G}}_{-}:=\left\{\hat{b}:=\sum_{j=0}^{\infty} \xi^{-(j+1)} b_{j}: b_{j} \in \tilde{\mathcal{G}}, j \in \mathbf{Z}_{+}\right\},
\end{aligned}
$$

are Lie subalgebras in $\hat{\mathcal{G}}$ and $\hat{\mathcal{G}}=\hat{\mathcal{G}}_{+} \oplus \hat{\mathcal{G}}_{-}$. Because of the splitting of $\hat{\mathcal{G}}$ into the direct, sum of its Lie subalgebras one can construct the so called Lie-Poisson structure $\frac{[37,38,39,40]}{37}$ on $\hat{\mathcal{G}}^{*}$, using a special linear endomorphism $\mathcal{R}$ of $\hat{\mathcal{G}}$ :

$$
\mathcal{R}:=\left(P_{+}-P_{-}\right) / 2, \quad P_{ \pm} \hat{\mathcal{G}}:=\hat{\mathcal{G}}_{ \pm}, \quad P_{ \pm} \hat{\mathcal{G}}_{\mp}=0 .
$$

For any smooth by Frechet functionals $\gamma, \mu \in \mathcal{D}\left(\hat{\mathcal{G}}^{*}\right)$ the Lie-Poisson bracket on $\hat{\mathcal{G}}^{*}$ is given by the expression:

$$
\{\gamma, \mu\}_{\mathcal{R}}(\hat{l})=\left(\hat{l},[\nabla \gamma(\hat{l}), \nabla \mu(\hat{l})]_{\mathcal{R}}\right)
$$

where $\hat{l} \in \hat{\mathcal{G}}^{*}$ and for all $\hat{a}, \hat{b} \in \hat{\mathcal{G}}$ the $\mathcal{R}$ commutator in (10.8) has the form $[39,40]$ :

$$
[\hat{a}, \hat{b}]_{\mathcal{R}}:=[\mathcal{R} \hat{a}, \hat{b}]+[\hat{a}, \mathcal{R} \hat{b}],
$$

subject to which the linear space $\hat{\mathcal{G}}$ becomes a Lie algebra too. The gradient $\nabla \gamma(\hat{l}) \in \hat{\mathcal{G}}$ of a functional $\gamma \in \mathcal{D}\left(\hat{\mathcal{G}}^{*}\right)$ at a point $\hat{l} \in \hat{\mathcal{G}}^{*}$ with respect to the scalar product (10.4) is defined as

$$
\delta \gamma(\hat{l}):=(\nabla \gamma(\hat{l}), \delta \hat{l})
$$

where the linear space isomorphism $\hat{\mathcal{G}} \simeq \hat{\mathcal{G}}^{*}$ is taken into account.

The Lie-Poisson bracket (10.8) generates Hamiltonian dynamical systems on $\hat{\mathcal{G}}^{*}$ related with Casimir invariants $\gamma \in I\left(\mathcal{G}^{*}\right)$, satisfying the condition:

$$
[\nabla \gamma(\hat{l}), \hat{l}]=0
$$

as the corresponding Hamiltonian functions. Due to the expressions (10.8) and (10.11)the mentioned above Hamiltonian system takes the form:

$$
d \hat{l} / d t:=[\mathcal{R} \nabla \gamma(\hat{l}), \hat{l}]=\left[\nabla \gamma_{+}(\hat{l}), \hat{l}\right]
$$


being equivalent to the usual commutator Lax type representation $\mathbb{P M}, \mathrm{La}, 41]$. The relationship (10.12) is a compatibility condition for the linear integral-differential equations:

$$
\begin{aligned}
\hat{l} f & =\lambda f, \\
d f / d t & =\nabla \gamma_{+}(\hat{l}) f,
\end{aligned}
$$

where $\lambda \in \mathbb{C}$ is a spectral parameter and a vector-function $f \in W\left(\mathbb{S}^{1} ; \mathbf{H}\right)$ is an element of some matrix representation for the Lie algebra $\hat{\mathcal{G}}$ in some functional Banach space $\mathbf{H}$.

Algebraic properties of the equation (10.12) together with (10.14) and the associated dynamical system on the space of adjoint functions $f^{*} \in W^{*}\left(\mathbb{S}^{1} ; \mathbf{H}\right)$ :

$$
d f^{*} / d t=-(\nabla \gamma(\hat{l}))_{+}^{*} f^{*}
$$

where $f^{*} \in W^{*}$ is a solution to the adjoint spectral problem:

$$
\hat{l}^{*} f^{*}=\nu f^{*},
$$

being considered as some coupled evolution equations on the space $\hat{\mathcal{G}}^{*} \oplus W \oplus W^{*}$ is an object of our further investigation.

2. The tensor product of Poisson structures and its Backlund transformation. To compactify the description below we will use the following designation of the gradient vector

$$
\nabla \gamma\left(\tilde{l}, \tilde{f}, \tilde{f}^{*}\right):=\left(\delta \gamma / \delta \tilde{l}, \delta \gamma / \delta \tilde{f}, \delta \gamma / \delta \tilde{f}^{*}\right)^{T}
$$

for any smooth functional $\gamma \in \mathcal{D}\left(\hat{\mathcal{G}}^{*} \oplus W_{\mathrm{B} 1} \oplus W_{\mathrm{S}}^{*}\right)_{\mathrm{PM}}$ On the spaces $\hat{\mathcal{G}}^{*}$ and $W \oplus W^{*}$ there exist canonical Poisson structures $\left[\frac{38,42,40]}{38,4}\right.$

$$
\delta \gamma / \delta \tilde{l}: \stackrel{\tilde{\theta}}{\rightarrow}\left[(\delta \gamma / \delta \tilde{l})_{+}, \tilde{l}\right]-[\delta \gamma / \delta \tilde{l}, \tilde{l}]_{+}
$$

at a point $\tilde{l} \in \hat{\mathcal{G}}^{*}$ and

$$
\left(\delta \gamma / \delta \tilde{f}, \delta \gamma / \delta \tilde{f}^{*}\right)^{T}: \stackrel{\tilde{J}}{\rightarrow}\left(\delta \gamma / \delta \tilde{f}^{*},-\delta \gamma / \delta \tilde{f}\right)^{T}
$$

at a point $\left(\tilde{f}, \tilde{f}^{*}\right) \in W \oplus W^{*}$ correspondingly. It should be noted that the Poisson structure (10.17) is transformed into (10.12) for any Casimir functional $\gamma \in I\left(\hat{\mathcal{G}}^{*}\right)$ - Thus, on the extended space $\hat{\mathcal{G}}^{*} \oplus W \oplus W^{*}$ one can obtain a Poisson structure as the tensor product $\tilde{\Theta}:=\tilde{\theta} \otimes \tilde{J}$ of the structures (10.17) and $(10.18)$.

Let us consider the following Backlund transformation $\frac{40,42,46] \text { : }}{40,4}$

$$
\left(\hat{l}, f, f^{*}\right): \stackrel{B}{\rightarrow}\left(\tilde{l}\left(\hat{l}, f, f^{*}\right), \tilde{f}=f, \tilde{f}^{*}=f^{*}\right),
$$


generating on $\hat{\mathcal{G}}^{*} \oplus W \oplus W^{*}$ a Poisson structure $\Theta$ with respect to variables $\left(\hat{l}, f, f^{*}\right)$ of the coupled evolution equations (10.12), (10.14), (10.15).

The main condition for the mapping (10.19) to be defined is the coincidence of the dynamical system

$$
\left(d \hat{l} / d t, d f / d t, d f^{*} / d t\right)^{T}:=-\Theta \nabla \gamma\left(\hat{l}, f, f^{*}\right)
$$

with $(10.12),(10.14),(10.15)$ in the case of $\gamma \in I\left(\hat{\mathcal{G}}^{*}\right)$, i.e. if this functional is taken to be not dependent of variables $\left(f, f^{*}\right) \in W \oplus W^{*}$. To satisfy that condition, one has to find a variation of any smooth Casimir functional $\gamma \in I\left(\hat{\mathcal{G}}^{*}\right)$ as $\delta \tilde{l}=0$, considered as a functional on $\hat{\mathcal{G}}^{*} \oplus W \oplus W^{*}$, taking into account flows (10.14), (10.15) and the Backlund transformation (10.19):

$$
\begin{aligned}
\left.\delta \gamma\left(\tilde{l}, \tilde{f}, \tilde{f}^{*}\right)\right|_{\delta \tilde{l}=0}=(<\delta \gamma / \delta \tilde{f}, \delta \tilde{f}>)+\left(<\delta \gamma / \delta \tilde{f}^{*}, \delta \tilde{f}^{*}>\right) & = \\
\left(<-d \tilde{f}^{*} / d t, \delta \tilde{f}>\right)+\left.\left(<d \tilde{f} / d t, \delta \tilde{f}^{*}>\right)\right|_{\tilde{f}=f, \tilde{f}^{*}=f^{*}} & = \\
\left(<(\delta \gamma / \delta \hat{l})_{+}^{*} f^{*}, \delta f>\right)+\left(<(\delta \gamma / \delta \hat{l})_{+} f, \delta f^{*}>\right) & = \\
\left(<f^{*},(\delta \gamma / \delta \hat{l})_{+} \delta f>\right)+\left(<(\delta \gamma / \delta \hat{l})_{+} f, \delta f^{*}>\right) & = \\
\left(\delta \gamma / \delta \hat{l}, \delta f \xi^{-1} \otimes f^{*}\right)+\left(\delta \gamma / \delta \hat{l}, f \xi^{-1} \otimes \delta f^{*}\right) & = \\
\left(\delta \gamma / \delta \hat{l}, \delta\left(f \xi^{-1} \otimes f^{*}\right)\right):=(\delta \gamma / \delta \hat{l}, \delta \hat{l}) . &
\end{aligned}
$$

As a result of the expression (10.21) one obtains the relationships:

$$
\left.\delta \hat{l}\right|_{\delta \tilde{l}=0}=\delta\left(f \xi^{-1} \otimes f^{*}\right),
$$

or having assumed the linear dependence of $\hat{l}$ and $\tilde{l} \in \hat{\mathcal{G}}^{*}$ one gets right away that

$$
\hat{l}=\tilde{l}+f \xi^{-1} \otimes f^{*} .
$$

Thus, the Backlund transformation (10.19) can be now written as

$$
\left(\hat{l}, f, f^{*}\right): \stackrel{B}{\rightarrow}\left(\tilde{l}=\hat{l}-f \xi^{-1} \otimes f^{*}, \quad \tilde{f}=f, \quad \tilde{f}^{*}=\tilde{f}^{*}\right) .
$$

The expression (10.24) generalizes the result, obtained in the papers $\left[\frac{\mathbb{M}, \mathrm{SP}}{40,46] \text { for }}\right.$ the Lie algebra $\hat{\mathcal{G}}$ of integral-differential operators with scalar coefficients. The existence of the Backlund transformation (10.19) makes it possible to formulate the following theorem.

Theorem 1. A dynamical system on $\hat{\mathcal{G}}^{*} \oplus W \oplus W^{*}$, being Hamiltonian with respect to the canonical Poisson structure $\tilde{\Theta}: T^{*}\left(\hat{\mathcal{G}}^{*} \oplus W \oplus W^{*}\right) \rightarrow T\left(\hat{\mathcal{G}}^{*} \oplus W \oplus W^{*}\right)$, and generated by the evolution equations:

$$
d \tilde{l} / d t=\left[\nabla \gamma_{+}(\tilde{l}), \tilde{l}\right]-[\nabla \gamma(\tilde{l}), \tilde{l}]_{+}, \quad d \tilde{f} / d t=\delta \gamma / \delta \tilde{f}^{*}, \quad d \tilde{f}^{*} / d t=-\delta \gamma / \delta \tilde{f},
$$


with $\gamma \in I\left(\mathcal{G}^{*}\right)$ being the Casimir functional at $\hat{l} \in \hat{\mathcal{G}}^{*}$ connected with $\tilde{l} \in \hat{\mathcal{G}}^{*}$ by (10.23), is equivalent to the system (10.12), (10.14) and (10.15) via the constructed above Backlund transformation (10.24).

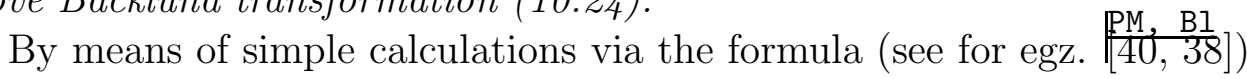

$$
\tilde{\Theta}=B^{\prime} \Theta B^{*}
$$

where $B^{\prime}: T\left(\hat{\mathcal{G}}^{*} \oplus W \oplus W^{*}\right) \rightarrow T\left(\hat{\mathcal{G}}^{*} \oplus W \oplus W^{*}\right)$ is the Frechet derivative of (24), one brings about the following form of the Poisson structure $\Theta$ on $\hat{\mathcal{G}}^{*} \oplus W \oplus W^{*} \ni$ $\left(\hat{l}, f, f^{*}\right)$ :

$\nabla \gamma\left(\hat{l}, f, f^{*}\right): \stackrel{\Theta}{\rightarrow}\left(\begin{array}{c}{\left[\hat{l},(\delta \gamma / \delta \hat{l})_{+}\right]-[\hat{l}, \delta \gamma / \delta \hat{l}]_{+}} \\ -f \xi^{-1} \otimes \delta \gamma / \delta f+\delta \gamma / \delta f^{*} \xi^{-1} \otimes f^{*} \delta \gamma / \delta f^{*}-(\delta \gamma / \delta \hat{l})_{+} f-\delta \gamma / \delta f+(\delta \gamma / \delta \hat{l})_{+}^{*} f\end{array}\right)$

that makes it possible to formulate the next theorem.

Theorem 2. The dynamical system (10.20), being Hamiltonian with respect to the Poisson structure $\Theta$ in the form (10.26) and a functional $\gamma \in I\left(\hat{\mathcal{G}}^{*}\right)$, gives the inherited Hamiltonian representation for the coupled evolution equations (10.12), (10.14), (10.15).

By means of the expression (10.23) one can construct Hamiltonian evolution equations, describing commutative flows on the extended space $\hat{\mathcal{G}}^{*} \oplus W \oplus W^{*}$ at a fixed element $\tilde{l} \in \hat{\mathcal{G}}^{*}$. Due to (10.24) every equation of such a type is equivalent to the system

$$
\left\{\begin{array}{l}
d \hat{l} / d \tau_{n}=\left[\hat{l}_{+}^{n}, \hat{l}\right] \\
d f / d \tau_{n}=\hat{l}_{+}^{n} f \\
d f^{*} / d \tau_{n}=-\left(\hat{l}^{*}\right)_{+}^{n} f^{*}
\end{array}\right.
$$

generated by involutive with respect to the Poisson bracket (10.17) Casimir invariants $\gamma_{n} \in I\left(\hat{\mathcal{G}}^{*}\right), n \in \mathbf{N}$, taking here the standard form:

$$
\gamma_{n}=1 /(n+1)\left(\hat{l}^{n}, \hat{l}\right)
$$

at $\hat{l} \in \hat{\mathcal{G}}^{*}$.

The compatibility conditions of the Hamiltonian systems (10.25) for different $n \in \mathbb{Z}_{+}$can be used for obtaining Lax integrable equations on usual spaces of smooth $2 \pi$-periodic multivariable functions that will be done in the next section.

3. The Lax type integrable Davey-Stewartson equation and its triple linear representation. Choose the element $\tilde{l} \in \hat{\mathcal{G}}^{*}$ in an exact form such as

$$
\tilde{l}=\left(\begin{array}{cc}
1 & 0 \\
0 & -1
\end{array}\right) \xi-\left(\begin{array}{cc}
0 & u \\
\bar{u} & 0
\end{array}\right)
$$


where $u, \bar{u} \in C^{\infty}\left(\mathbb{S}^{1} ; \mathbb{C}\right)$ and $\mathcal{G}=g l(2 ; \mathbb{C})$. Then

$$
\hat{l}=\tilde{l}+\left(\begin{array}{cc}
f_{1} \xi^{-1} f_{1}^{*} & f_{1} \xi^{-1} f_{2}^{*}+u \\
\bar{u}+f_{2} \xi^{-1} f_{1}^{*} & f_{2} \xi^{-1} f_{2}^{*}
\end{array}\right)
$$

where $f=\left(f_{1}, f_{2}\right)^{T}$ and $f^{*}=\left(f_{1}^{*}, f_{2}^{*}\right)^{T}$, "- " can sign the complex conjugation. Below we will study the evolutions (10.25) of vector-functions $\left(f, f^{*}\right) \in$ $W\left(\mathbb{S}^{1} ; \mathbb{C}^{2}\right) \oplus W^{*}\left(\mathbb{S}^{1} ; \mathbb{C}^{2}\right)$ with respect to the variables $y=\tau_{1}$ and $t=\tau_{2}$ at the point (10.26). They can be obtained from the second and third equations in (10.25), having put $n=1$ and $n=2$, as well as from the first one. The latter is the compatibility condition of the spectral problem

$$
\hat{l} \Phi=\lambda \Phi,
$$

where $\Phi=\left(\Phi_{1}, \Phi_{2}\right)^{T} \in W\left(\mathbb{S}^{1} ; \mathbb{C}^{2}\right), \lambda \in \mathbb{C}$ is some parameter, with the following linear equations:

$$
\begin{aligned}
d \Phi / d y & =\hat{l}_{+} \Phi \\
d \Phi / d t & =\hat{l}_{+}^{2} \Phi
\end{aligned}
$$

arising from (10.26) at $n=1$ and $n=2$ correspondingly. The compatibility of equations (10.28) and (10.29) leads to the relationships:

$$
\begin{aligned}
& \partial u / \partial y=-2 f_{1} f_{2}^{*}, \quad \partial \bar{u} / \partial y=-2 f_{1}^{*} f_{2}, \\
& \partial f_{1} / \partial y=\partial f_{1} / \partial x-u f_{2}, \quad \partial f_{1}^{*} / \partial y=\partial f_{1}^{*} / \partial x-\bar{u} f_{2}^{*}, \\
& \partial f_{2} / \partial y=-\partial f_{2} / \partial x+\bar{u} f_{1}, \quad \partial f_{2}^{*} / \partial y=-\partial f_{2}^{*} / \partial x+u f_{1}^{*}
\end{aligned}
$$

Analogously, replacing $t \in \mathbb{C}$ by $i t \in i \mathbb{R}, i^{2}=-1$, one gets from (10.29) and (10.30):

$$
\begin{gathered}
d u / d t=i\left(\partial^{2} u / \partial x \partial y+2 u\left(f_{1} f_{1}^{*}+f_{2} f_{2}^{*}\right)\right), \quad d \bar{u} / d t=-i\left(\partial^{2} \bar{u} / \partial x \partial y+2 \bar{u}\left(f_{1} f_{1}^{*}+f_{2} f_{2}^{*}\right)\right), \\
\partial\left(f_{1} f_{1}^{*}\right) / \partial y-\partial\left(f_{1} f_{1}^{*}\right) / \partial x=1 / 2 \partial(u \bar{u}) / \partial y=-\left(\partial\left(f_{2} f_{2}^{*}\right) / \partial x+\partial\left(f_{2} f_{2}^{*}\right) / \partial y\right), \\
d f_{1} / d t=i\left(\partial^{2} f_{1} / \partial x^{2}+\left(2 f_{1} f_{1}^{*}-u \bar{u}\right) f_{1}-\partial u / \partial x f_{2}\right), \\
d f_{1}^{*} / d t=-i\left(\partial^{2} f_{1}^{*} / \partial x^{2}+\left(2 f_{1} f_{1}^{*}-u \bar{u}\right) f_{1}^{*}-\partial \bar{u} / \partial x f_{2}^{*}\right), \\
d f_{2} / d t=i\left(\partial^{2} f_{2} / \partial x^{2}-\left(2 f_{2} f_{2}^{*}+u \bar{u}\right) f_{2}-\partial \bar{u} / \partial x f_{1}\right), \\
d f_{2}^{*} / d t=-i\left(\partial^{2} f_{2}^{*} / \partial x^{2}-\left(2 f_{2} f_{2}^{*}+u \bar{u}\right) f_{2}^{*}-\partial u / \partial x f_{1}^{*}\right) .
\end{gathered}
$$

The relationships (10.31), (10.32) take the well known form of the Davey-Stewartson equation $\left[\frac{38,43]}{38}\right.$ at $\bar{u} \in \mathrm{C}^{\infty}\left(\mathbb{S}^{1} ; \mathbb{C}\right)$ being a complex conjugated to $u \in \mathrm{C}^{\infty}\left(\mathbb{S}^{1} ; \mathbb{C}\right)$. The compatibility for every pair of equations (10.28), (10.29) and (10.30), which 
can be rewritten as the first order linear ordinary differential ones in such a way:

$$
\begin{aligned}
& d \Phi / d x=\left(\begin{array}{ccc}
\lambda & u & -f_{1} \\
\bar{u} & -\lambda & f_{2} \\
f_{1}^{*} & f_{2}^{*} & 0
\end{array}\right) \Phi, \\
& d \Phi / d y=\left(\begin{array}{ccc}
\lambda & 0 & -f_{1} \\
0 & \lambda & -f_{2} \\
f_{1}^{*} & f_{2}^{*} & 0
\end{array}\right) \Phi, \\
& d \Phi / d t=i\left(\begin{array}{ccc}
\lambda^{2}+f_{1} f_{1}^{*} & 1 / 2 \partial u / \partial y & -\lambda f_{1}-\partial f_{1} / \partial y \\
-1 / 2 \partial \bar{u} / \partial y & \lambda^{2}-f_{2} f_{2}^{*} & -\lambda f_{2}-\partial f_{1} / \partial y \\
\lambda f_{1}^{*}+\partial f_{1}^{*} / \partial y & \lambda f_{2}^{*}+\partial f_{2}^{*} / \partial y & 0
\end{array}\right) \Phi(10.34)
\end{aligned}
$$

where $\Phi=\left(\Phi_{1}, \Phi_{2}, \Phi_{3}\right)^{T} \in W\left(\mathbb{S}^{1} ; \mathbb{C}^{3}\right)$, provide its Lax type integrability. Thus, the following theorem holds.

Theorem 3. The Davey-Stewartson equation (10.32), (10.33) possesses the Lax representation as the compatibility condition for equations (10.34) under the additional natural constraint (10.27).

In fact, one has found above a triple linearization for a $(2+1)$-dimensional dynamical system, that is a new important ingredient of the Lie algebraic approach to Lax type integrable flows, based on the Backlund type transformation (10.23) developed in this work. It is clear that the similar construction of a triple linearization like (10.4) can be done for many other both old and new $(2+1)$-dimensional dynamical systems, on what we plant to stop in detail in another work under preparation.

\section{Conclusion}

The developed above theory of parametrically Lax-type integrable dynamical systems concedes to widen to a great extent the class of exactly treated nonlinear models in many fields of science. It is to be noted here the following important mathematical fact being got in the paper: almost every nonlinear dynamical system admits a parametrically isospectral Lax type representation but a given dynamical system is the Lax-type integrable if an evolution of the spectrum parameter doesn't depend on a point $u \in M$ at all Cauchy data. This result has allowed us to develop a very effective direct criterion for the following problem: whether a given nonlinear dynamical system on the functional manifold $M$ is parametrically Lax-type integrable or not. Having the problem above solved, we have suggested the reduction procedure for the associated nonlinear dynamical systems to be descended on the invariant submanifold $M_{N} \subset M$ built before inheriting the canonical Hamiltonian structure and the Liouville complete integrability. Thereby, 
the powerful techniques of perturbation theory can be successfully used for dynamical systems under consideration, as well as the relationships between the full Hamiltonian theory and various Hamiltonian truncations could be now got understandable more deeply.

The imbedding problem for infinite-dimensional dynamical systems with additional structures such as invariants and symmetries is as old as the NewtonLagrange mechanics, having been treated by many researches, using both analytical and algebraic methods. The powerful differential-geometric tools used here were created mainly in works by E. Cartan at the beginning of the twentieth century. The great impact in the development of imbedding methods was done in last time, especially owing to theory of isospectral deformations for some linear structures built on the special vector bundles over the spase $M$ as the base of a given nonlinear dynamical system. Among them there are such structures as the moment map $l: M \rightarrow \mathcal{G}^{*}$ into the adjoint space to the Lie algebra $\mathcal{G}$ of symmetries, acting on the symplectic phase space $M$ equivariantly $\left[\frac{1}{8,10}\right]$, the connection of the Cartan-Eresman structures appearing via the Wahlquist-Estabrook approach [II], and many others.

For the last years the general structure of Lagrangian and Hamiltonian formalisms was studied thoroughly using both geometrical and algebraical methods [12, 13]. The special attention was paid to the theory of differential-difference dy-

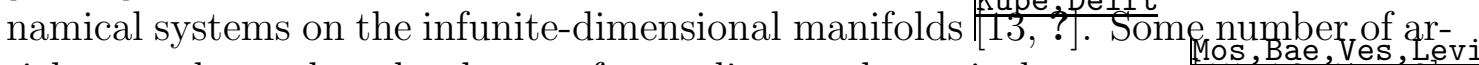
ticles was devoted to the theory of pure discrete dynamical systems $\frac{\text { Mos, } 16,16,17,18],}{15,16}$ as well treating the interesting examples $\frac{\| \text { [18] }}{[18}$ appeared to be important for applications.

In future work we intend to treat further imbedding problems for infinitedimensional both continuous and discrete dynamical systems basing on the differentialgeometric Cartan's theory of differential ideals in Grassmann algebras over jetmanifolds, intimately connected with the problem under regard. As it is well knowp there existed by now only two regular enough algorithmic approaches $[36,40,46]$ to constructing integrable multi-dimensional (mainly 2+1) dynamical systems on infinite-dimensional functional spaces. Our approach ${ }_{\mathbb{P M}}$ deysised in

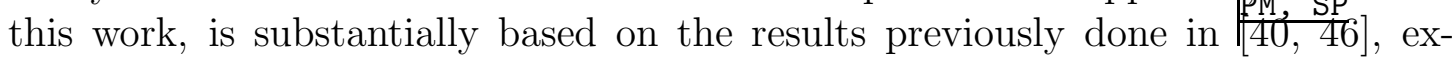
plains completely the computational properties of multi-dimensional flows before delivered in works $[38,43]$. As the key points of our approach there used the canonical Hamiltonian structures naturally existing on the extended phase space and the related with them Backlund transformation which saves Casimir invariants of a chosen matrix integral-differential Lie algebra. The latter gives rise to some additional Hamiltonian properties of considered extended evolution flows before studied in $[38,40]$ making use of the standard inverse scattering transform $[41,38,40]$ and the formal symmetry reduction for the KP-ierarchy $[42,46]$ of 
commuting operator flows.

As one can convince ourselves analyzing the structure of the Backlund type transformation (10.24), that it strongly depends on the type of an ad-invariant scalar product chosen on an operator Lie algebra $\hat{\mathcal{G}}$ and its Lie algebra decomposition like (10.6). Since there exist in general other possibilities of choosing such decompositions and $a d$-invariant scalar products on $\hat{\mathcal{G}}$, they give rise naturally to another resulting types of the corresponding Backlund transformations, which can be a subject of another special investigation. Let us here only mention the choice of a scalar product related with the operator Lie algebra $\hat{\mathcal{G}}$ centrally extended by means of the standard Maurer-Cartan two-cocycle [36, 39, 40], bringing about new types of multi-dimensional integrable flows.

The last aspect of the Backlund approach to constructing Lax type integrable flows and their partial solutions which is worth of mention is related with DarbouxBacklund type transformations $\left[\frac{38,44]}{38}\right.$ and their new generalization recently developed in $[45,46]$. They give rise to very effective procedures of constructing multi-dimensional integrable flows on functional spaces with arbitrary number of independent variables simultaniously delivering a wide class of their exact analytical solutions, depending on many constant parameters, which can appear to be useful for diverse applications in applied sciences.

All mentioned above Backlund type transformations aspects can be studied as special investigations, giving rise to new directions in the theory of multidimensional evolution flows and their integrability.

\section{Acknowledgements}

The authors thank Profs. D.L. Blackmore (NJIT, Newark, NJ,USA), M.O. Perestiuk (Kyiv National University, Ukraina) for useful comments on the results of the work.

\section{References}

Grif [1] P. A. Griffiths. Exterior Differential Systems and the Calculus of Variations. New York. Birkhause, 1982, 480p.

Fil [2] Prykarpatsky A. K., B. M. Fil. Category of topological jet-manifolds and certain applications in the theory of nonlinear infinite-dimensional dynamical systems. // Ukr. Math. J., 1993, vol. 44, No 2 p. 1136-1147. 
Lax [3] P. D. Lax. Periodic solutions of the Korteweg-de Vries equation. // Comm. Pure and Appl. Mathem., 1968, vol. 21, No 2, p. 467-490.

Bry [4] R. L. Bryant. On notions of Equivalence of Variational Problems with One Independent Variable. // Contemp. Math., 1987, vol. 63, p. 65-76.

St [5] S. Sternberg. Some preliminary remarks on the formal variational calculus of Gel'fand and Dikii. // Lect. Notes in Mathem., 1980, vol. 150, p. 399-407.

Stern [6] S. Sternberg. Lectures on differential geometry. Moscow, Mir, 1961, p. 412.

Bogo [7] O. Bogoyavlensky, S. Novikov. On connection of Hamiltonian formalisms of stationar and nonstationar problems. // Function. Anal.

Pri [8] Prykarpatsky A. K., I. V. Mykytiuk. Algebraic aspects of integrable dynamical systems on manifolds. Kiev, Nauk. dumka, 1991, 380p.

Gelf [9] I. M. Gelfand, L. A. Dikiy Integrable nonlinear equations and Liouville theorem. // Function. Anal. Appl., 1979, vol. 13, No 1, p. 8-20.

Gill [10] V.Gillemin, S.Sternberg. The moment map and Collective motion. // Ann. Phys., 1980, vol. 127, No 2, p. 220-253

Wah [11] Wahlgnist H. D., Estabrook F. B. Prolongation structures of nonlinear evolution equations. // J. Math. Phys., 1975, vol. 16, No 1, p. 1-7, 1976, vol. 17, No 7, p. 1293-1297.

Kup [12] Kupershmidt B. A. Geometry of jet-bundles and the structure of Lagrangian and Hammmiltonian formalisms. // Lect. Notes Math., 1980, vol. 775, p. $162-218$.

Kupe [13] Kupershmidt B. A. Discrete Lax equations and differential-difference calculus. // Asterisque, 1985, vol. 123, p. 5-212.

Duft [14] Deift P. Li L.-C., Tomei C. Loop groups, discrete versions of some classical integrable systems and rank-2 extensions. // Memoirs of the AMS, 1992, vol. 100, No 479, p. 1-101

Mos [15] Moser J., Veselov A. P. Discrete versions of some classical integrable systems and factorization of matrix polynomials. Zurich, ETH, Preprint, 1989, 76p.

Bae [16] Baez J. C., Gillam J. W. An algebraic approach to discrete mechanics. // Lett. Math. Phys., 1994, vol. 31, No 3, p. 205-212.

Ves [17] Veselov A. P. What is an itegrable mapping? in: What is itegrability? Ney York, Springer-Verlag, 1991, p. 251-272. 
Levi [18] Levi D., Winternitz P. Continuous symmetries of discrete equations. // Physics Lett. A., 1991, vol. 152, No 7, p. 335-338.

Kan [19] Kaneko K. Symplectic cellular automate. // Phys. Lett. A., 1988, vol. 129, No 1, p. 9-16.

Mars [20] Albraham R., Marsden J. Foundation of mechanics. London, The Blujamin Publish. Co., 1978, p. 806.

Sou [21] Souriau J. M. Structure des systemes dynamique. Paris, Dunod, 1970.

Adl [22] Adler M. On a trace functional for formal pseudo-differential operators and the symplectic structures of the Korteweg-de Vries equations. // Invent. Mathem., 1979, vol. 50, No 2, p. 219-248.

Pry [23] Prykarpatsky A.K. and others. Algebraic structure of the gradient-golonomic algorithm for Lax integrable nonlinear dynamical systems. // J. Math. Phys., vol. 35, No 4, p. 1763-1777, vol. 35, No 8, p. 6115-6126.

Dew [24] Oewel W. Dirac constraints in field theory: Lifts of Hamiltonian systems to the cotangent bundle. // J. Math. Phys., 1988, vol. 29, No 1, p. 210-219.

De [25] Oewel W. R-structures, Yang-Baxter equations and related involution theorems. // J. Math. Phys., 1989, vol. 30, No 5, p. 1140-1149.

Fok [26] Fokas A. S., Gelfand I. M. Bi-Hamiltonian Structures and Integrability. in: Important developments in Soliton Theory. Springer-Verlag, 1992.

0lv [27] Olver P. J. Canonical forms and integrability of bi-Hamiltonian systems. // Phys. Lett. A., 1990, vol. 148, No 3, p. 177-187

Mag [28] Magri F. A simple model of the integrable Hamiltonian equation. // J. Math. Phys., 1978, vol. 19, No 3, p. 1156-1162.

Fer [29] Fernandes R. L. Completely integrable bi-Hamiltonian systems. // J. of Dynam. and Diff. Equ., 1994, vol. 6, No 1, p. 53-69.

Mit [30] Mitropolsky Yu. A., Bogoliubov N. N., Prykarpatsky A. K., Samoilenko V.H. Integrable dynamical systems. Spectral and differential-geometric aspects. Kiev, Naukova Dumka, 1987.

Nov [31] S.P.Novikov (editor). The theory of solitons. Moscov, Mir, 1980. 
Prik [32] Mitropolsky Yu. O., Prykarpatsky A. K., Fil B. M. Some aspects of a gradientholonomic algorithm in the theory of integrability of nonlinear dynamical systems and computer algebra problems // Ukrainian Math. J., 1991, vol. 43, No 1, p. 63-74.

Kuy [33] Kuybida V. V., Prytula M. M., Prykarpatsky A. K. The study of properties of the parametric isospectral integrability of nonlinear dynamical systems on functional manifolds and their finitedimensional approximations. Preprint of Inst. for Appl. Problems of Mech. and Mathem. of Ukr. Academy of Sci., Lviv, 1991, No 10, 41p.

Wein [34] Marsden J., Weinstein A. Reduction of symplectic manifolds with symmetries. // Rep. Math. Phys., 1974, vol. 5, No 2, p. 121-130.

Str [35] Oewel W., Strampp W. Constrained KP-hierarchy and bi-Hamiltonian structures. // Comm. Math. Phys., 1993, vol. 157, No 1, p. 51-81.

PSA [36] Prykarpatsky A.K., Samoilenko V.Hr., Andrushkiw R.I., Mitropolsky Yu.O., Prytula M.M.\} Algebraic structure of the gradient-holonomic algorithm for Lax integrable nonlinear systems. I // J. Math. Phys. - 1999. - 35, 2116 4. 421. 1763-1777.

Ad [37] Adler M. On a trace functional for formal pseudo-differential operators and the symplectic structures of a Korteweg-de Vries equation // Invent. Math. 1979. - 50, 2116 2. - P. 219-248.

B1 [38] Blaszak M. Multi-Hamiltonian theory of dynamical systems. - Verlag-BerlinHeidelberg: Springer, 1998. - 345 p.

De [39] Oevel W. R-structures, Yang-Baxter equations and related involution theorems // J. Math. Phys. - 1989. - 30, 2116 5. - P. 1140-1149.

PM [40] Prykarpatsky A.K., Mykytiuk I.V. Algebraic integrability of nonlinear dynamical systems on manifolds: classical and quantum aspects. - DordrechtBoston-London: Kluwer Academic Publishers, 1998. - 553 p.

La [41] Lax P.D. Periodic solutions of the KdV equation // Commun. Pure and Appl. Math. - 1975. - 28. - 421. 141-188.

OS [42] Oevel W., Strampp W. Constrained KP hierarchy and bi-Hamiltonian structures // Commun. Math. Phys. - 1993. - 157. - P. 51-81.

KSS [43] Konopelchenko B., Sidorenko Yu., Strampp W. (1+1)-dimensional integrable systems as symmetry constraints of (2+1)-dimensional systems // Phys. Lett. A. $-1991 .-157 .-421.17-21$. 
MS [44] Matveev V.B., Salle M.I. Darboux-Backlund transformations and applications. - New York: Springer, 1993.

Ni [45] Nimmo J.C.C. In: Nonlinear evolution equations and dynamical systems (NEEDS'94) / Ed. by Makhankov V.G., Bishop A.R. and Holm D.D. - World Scient. Publ., 1994.

SP [46] Samoilenko A.M., Prykarpatsky Ya.A. Algebraic-analytic aaspects of integrable nonlinear dynamical systems and their perturbations. - Kyiv: Intitute of Mathematics at NAS of Ukraine, 2002 